\title{
Aromaticity and Curvature in Heteroacepentalenes
}

\author{
Mark Mascal
}

\section{Table of Contents}

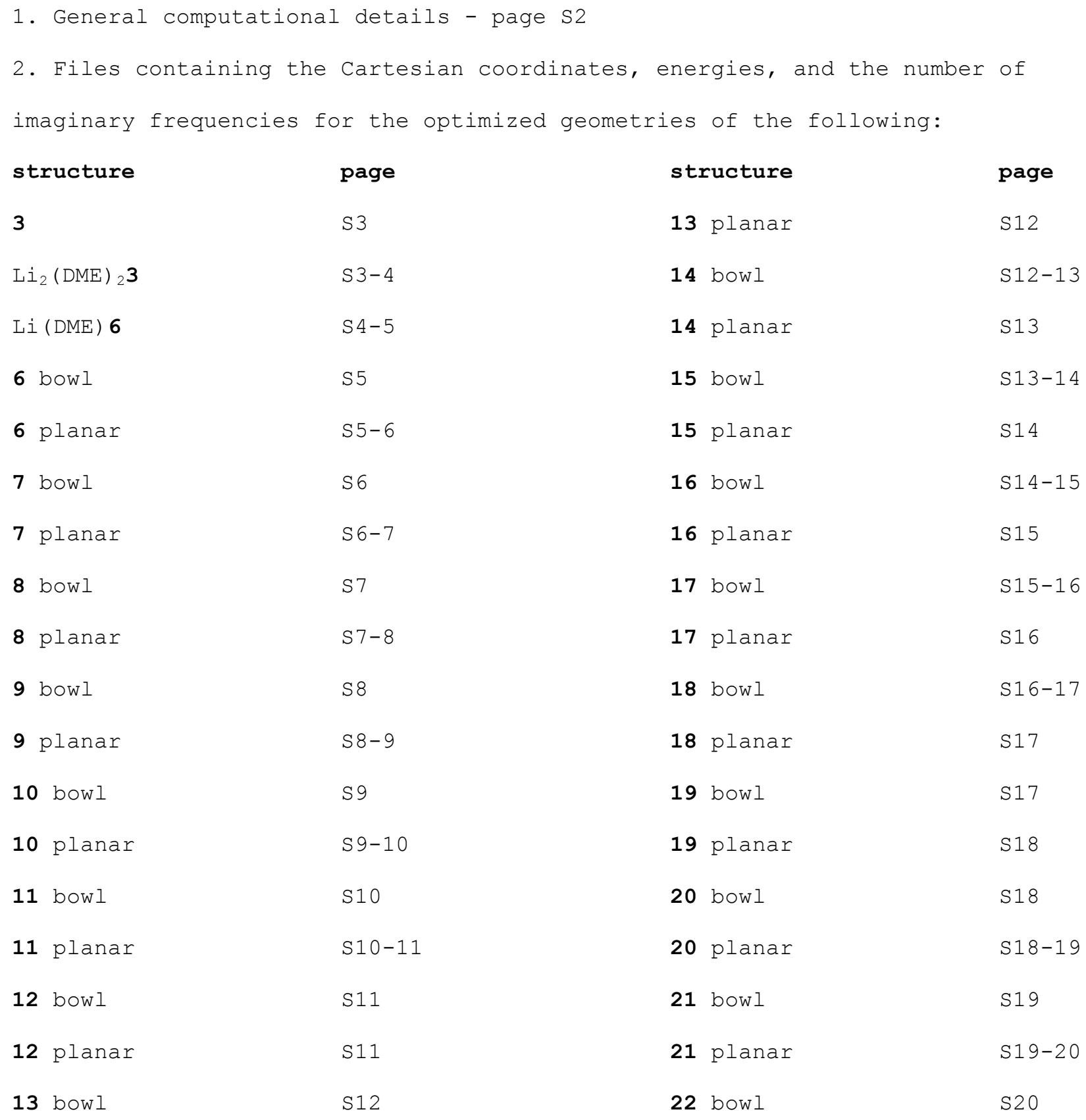




$\begin{array}{llll}\text { structure } & \text { page } & \text { structure } & \text { page } \\ \mathbf{2 2} \text { planar } & \mathrm{S} 20-21 & \mathbf{3 1} & \mathrm{S} 27-28 \\ \mathbf{2 3} \text { bowl } & \mathrm{S} 21 & \mathbf{3 2} & \mathrm{S} 28 \\ \mathbf{2 3} \text { planar } & \mathrm{S} 21-22 & \mathbf{3 3} & \mathrm{S} 28 \\ \mathbf{2 4} \text { bowl } & \mathrm{S} 22 & \mathbf{3 4} & \mathrm{S} 29 \\ \mathbf{2 5} \text { bowl } & \mathrm{S} 22 & \mathbf{3 5} & \mathrm{S} 29 \\ \mathbf{2 5} \text { planar } & \mathrm{S} 22-23 & \mathbf{3 6} \text { bowl } & \mathrm{S} 30 \\ \mathbf{2 6} \text { bowl } & \mathrm{S} 23 & \mathbf{3 6} \text { planar } & \mathrm{S} 30 \\ \mathbf{2 6} \text { planar } & \mathrm{S} 23-24 & \mathbf{3 7} \text { bowl } & \mathrm{S} 31 \\ \mathbf{2 7} \text { bowl } & \mathrm{S} 24 & \mathbf{3 7} \text { planar } & \mathrm{S} 31 \\ \mathbf{2 7} \text { planar } & \mathrm{S} 24-25 & & \\ \mathbf{2 8} \text { bowl } & \mathrm{S} 25 & \mathrm{~S} 25-26 & \\ \mathbf{2 8} \text { planar } & \mathrm{S} 26 & & \\ \mathbf{2 9} & \mathrm{S} 27 & & \\ \mathbf{3 0} & \mathrm{S} & & \end{array}$

General computational details: All calculations were performed using the following Gaussian command line:

\# opt freq=noraman rb3lyp/6-311++g $(d, p)$ symm=loose

The calculations were run in Gaussian 03:RevB.04 mounted in a multiprocessor PC under the Linux operating system. Default SCF convergence criteria were used. Energies are given in Hartrees. GIAO NMR calculations were performed using the following Gaussian command line:

\# nmr rb3lyp/6-311++g $(d, p)$ 
Data for Structure 3

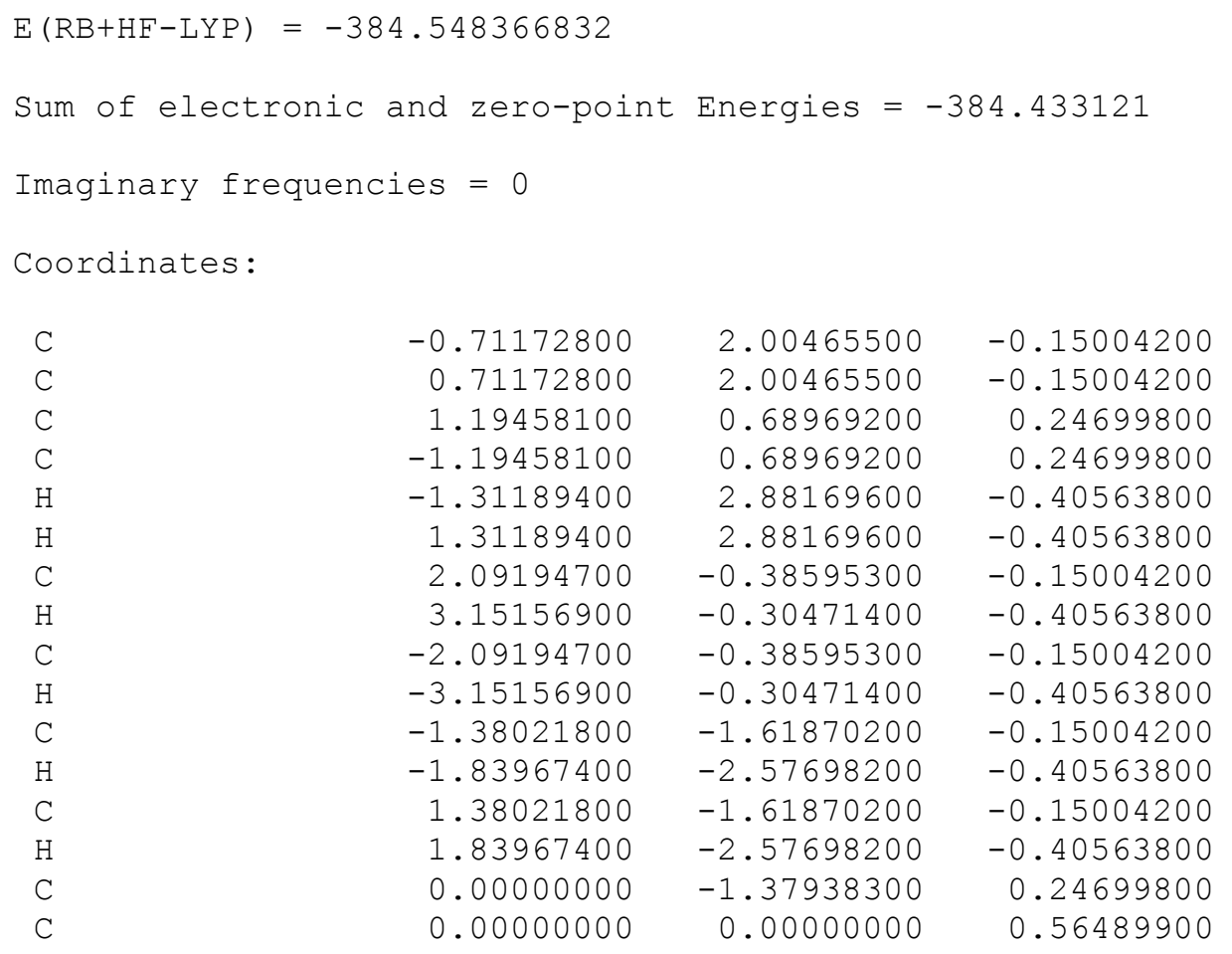

Data for Structure $\mathrm{Li}_{2}(\mathrm{DME})_{2} 3$

\author{
$\mathrm{E}(\mathrm{RB}+\mathrm{HF}-\mathrm{LYP})=-1017.75348655$ \\ Coordinates:
}

$$
\begin{array}{rr}
-1.57622700 & 0.40425400 \\
-1.22301100 & 1.75534900 \\
-1.85804100 & 2.47978500 \\
0.12396500 & 2.04474000 \\
0.59800400 & 3.00590100 \\
0.68565200 & 0.88332300 \\
1.79801100 & -0.02703500 \\
2.84311400 & 0.23095200 \\
1.30011300 & -1.37374500 \\
1.93635200 & -2.23620300 \\
-0.13585100 & -1.36609700 \\
-1.32751900 & -1.93897700 \\
-1.56969000 & -2.99063200 \\
-2.17115000 & -0.89294000 \\
-3.12857900 & -1.07455300 \\
-0.42100000 & 0.01123100 \\
-2.15276700 & 0.86641900 \\
-2.57771200 & 0.50939200 \\
-2.81540200 & 0.60786200 \\
-2.03001700 & 1.95487700 \\
-0.90813200 & 0.22194000 \\
0.07991900 & 0.46758400 \\
-0.30990100 & 0.24756000
\end{array}
$$




$\begin{array}{lrrr}\mathrm{H} & 4.82802000 & 0.38987700 & 1.52139200 \\ \mathrm{C} & 4.55691100 & 1.24757000 & -0.44489400 \\ \mathrm{H} & 5.22187500 & 2.08888300 & -0.20688400 \\ \mathrm{H} & 4.71198900 & 0.96311700 & -1.49423200 \\ \mathrm{O} & 3.19803200 & 1.61632900 & -0.24464300 \\ \mathrm{C} & 2.82425300 & 2.78571800 & -0.97535300 \\ \mathrm{H} & 3.40003700 & 3.65060500 & -0.62433100 \\ \mathrm{H} & 1.76206400 & 2.93745400 & -0.79974200 \\ \mathrm{H} & 2.99875600 & 2.64120600 & -2.04798300 \\ \mathrm{C} & -4.16187700 & 2.54954200 & 0.81699200 \\ \mathrm{H} & -3.20370600 & 2.98981100 & 1.08741900 \\ \mathrm{H} & -4.88403200 & 2.70877600 & 1.62621000 \\ \mathrm{H} & -4.53372900 & 3.01955000 & -0.10151700 \\ \mathrm{O} & -3.93235600 & 1.15809300 & 0.61935800 \\ \mathrm{C} & -5.09126700 & 0.42510000 & 0.23253400 \\ \mathrm{H} & -5.42801200 & 0.76064300 & -0.75768200 \\ \mathrm{H} & -5.90450400 & 0.58210600 & 0.95315500 \\ \mathrm{C} & -4.70401100 & -1.03932000 & 0.19847600 \\ \mathrm{H} & -4.49026900 & -1.40354300 & 1.21270100 \\ \mathrm{H} & -5.52762200 & -1.63384500 & -0.22010400 \\ \mathrm{O} & -3.54138100 & -1.14392800 & -0.60798000 \\ \mathrm{C} & -3.04115700 & -2.47952600 & -0.75075300 \\ \mathrm{H} & -2.11185500 & -2.40784600 & -1.31348200 \\ \mathrm{H} & -3.77071100 & -3.09658200 & -1.28820200 \\ \mathrm{H} & -2.83266200 & -2.91671800 & 0.23098600 \\ \mathrm{Li} & 1.95375200 & -0.00197300 & 0.23064900 \\ \mathrm{Li} & -2.22153200 & 0.35002800 & -0.19354700\end{array}$

\section{Data for Structure Li (DME) 6}

$E(R B+H F-L Y P)=-717.900440313$

Coordinates:

$$
\begin{array}{r}
-3.26378400 \\
-2.57534200 \\
-3.40621800 \\
-2.56005600 \\
-1.35926100 \\
-0.50238100 \\
-4.06822200 \\
-2.53217400 \\
-3.68228000 \\
-4.26133500 \\
-3.78233100 \\
-3.10459400 \\
-2.32110600 \\
-0.99185600 \\
0.41292200 \\
-0.26378500 \\
-0.67382000 \\
0.56565100 \\
1.08334700 \\
0.78655400 \\
1.68704000 \\
1.46918800
\end{array}
$$

$$
\begin{array}{r}
-0.15678900 \\
0.15999600 \\
0.14546200 \\
0.61120000 \\
-0.15296300 \\
0.08410600 \\
0.56431400 \\
-0.09932000 \\
-1.16903000 \\
0.82009300 \\
-0.87158800 \\
0.45600100 \\
1.67846200 \\
-0.25873100 \\
-0.47793100 \\
1.14929700 \\
-0.39186700 \\
-0.61642300 \\
0.53371300 \\
-1.77637400 \\
1.83771600 \\
-0.00218000
\end{array}
$$




$\begin{array}{lrrr}\mathrm{C} & 1.46163100 & -0.15543300 & -1.37195300 \\ \mathrm{C} & 2.48040300 & -0.27651800 & 1.92646400 \\ \mathrm{C} & 2.39005700 & 0.45228000 & 0.69601500 \\ \mathrm{C} & 2.37188600 & 0.93738000 & -1.57593100 \\ \mathrm{C} & 2.93840700 & 1.29113400 & -0.32568300 \\ \mathrm{H} & 0.12871300 & -3.15846300 & -0.63790100 \\ \mathrm{H} & 0.53434300 & -1.64169400 & -2.78708000 \\ \mathrm{H} & 1.57890300 & -2.18046000 & 2.62009700 \\ \mathrm{H} & 3.07417800 & -0.00493500 & 2.78747800 \\ \mathrm{H} & 2.61314200 & 1.40258400 & -2.52032000 \\ \mathrm{H} & 3.68423600 & 2.06113100 & -0.18838200\end{array}$

\section{Data for Structure 6 (bowl conformer)}

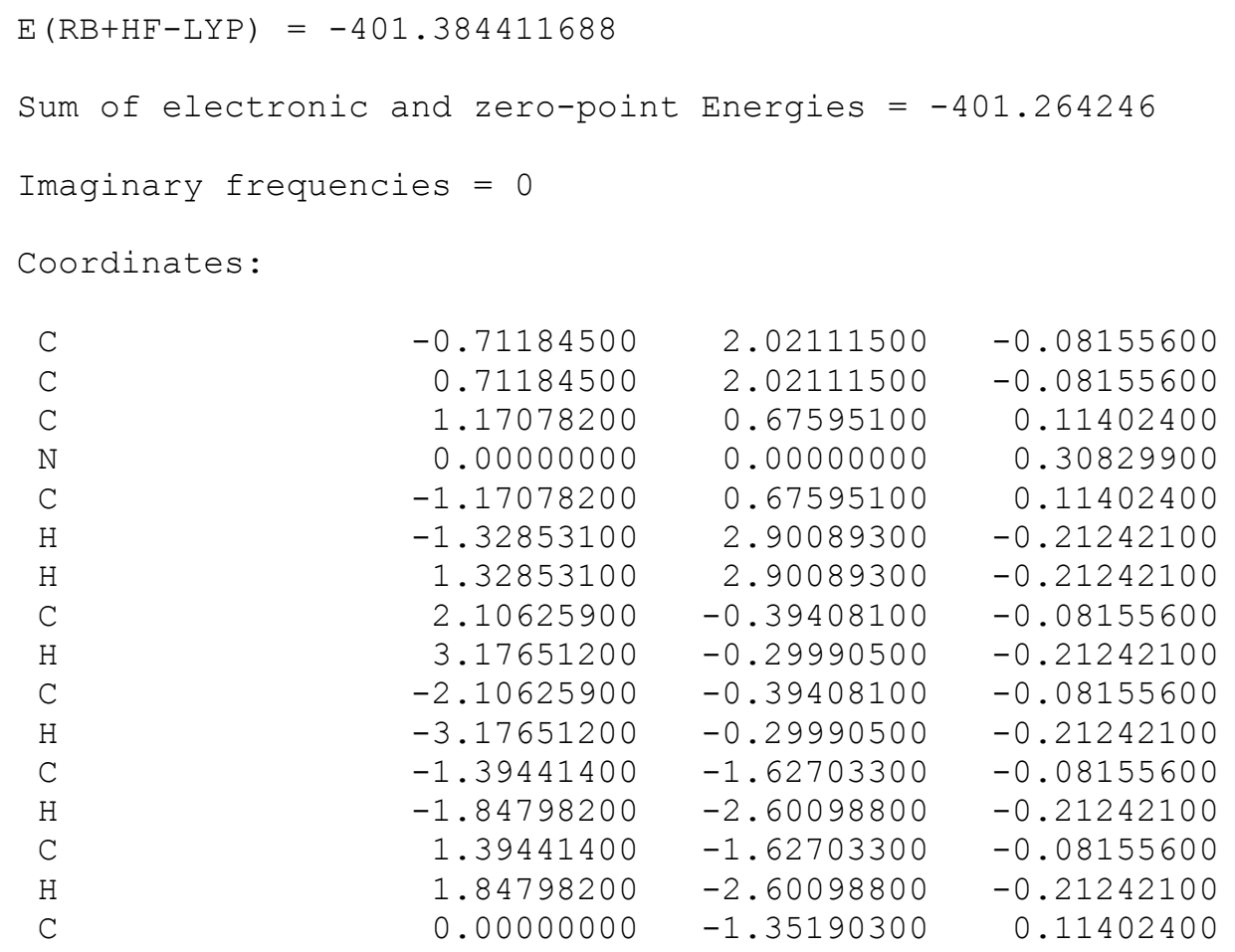

\section{Data for Structure 6 (planar conformer)}

$E(R B+H F-L Y P)=-401.383466576$

Sum of electronic and zero-point Energies $=-401.263729$

Imaginary frequencies $=1$

Coordinates:

$\begin{array}{lrrr}\text { C } & 2.12414700 & 0.40187400 & 0.00000000 \\ \mathrm{C} & 1.41010600 & 1.63862900 & 0.00000000 \\ \mathrm{C} & 0.00000000 & 1.35484000 & 0.00000000 \\ \mathrm{~N} & 0.00000000 & 0.00000000 & 0.00000000 \\ \mathrm{C} & 1.17332600 & -0.67742000 & 0.00000000 \\ \mathrm{H} & 3.20260900 & 0.31457700 & 0.00000000\end{array}$




$\begin{array}{lrrr}\mathrm{H} & 1.87373600 & 2.61625300 & 0.00000000 \\ \mathrm{C} & -1.41010600 & 1.63862900 & 0.00000000 \\ \mathrm{H} & -1.87373600 & 2.61625300 & 0.00000000 \\ \mathrm{C} & 0.71404100 & -2.04050200 & 0.00000000 \\ \mathrm{H} & 1.32887300 & -2.93083000 & 0.00000000 \\ \mathrm{C} & -0.71404100 & -2.04050200 & 0.00000000 \\ \mathrm{H} & -1.32887300 & -2.93083000 & 0.00000000 \\ \mathrm{C} & -2.12414700 & 0.40187400 & 0.00000000 \\ \mathrm{H} & -3.20260900 & 0.31457700 & 0.00000000 \\ \mathrm{C} & -1.17332600 & -0.67742000 & 0.00000000\end{array}$

\section{Data for Structure 7 (bowl conformer)}

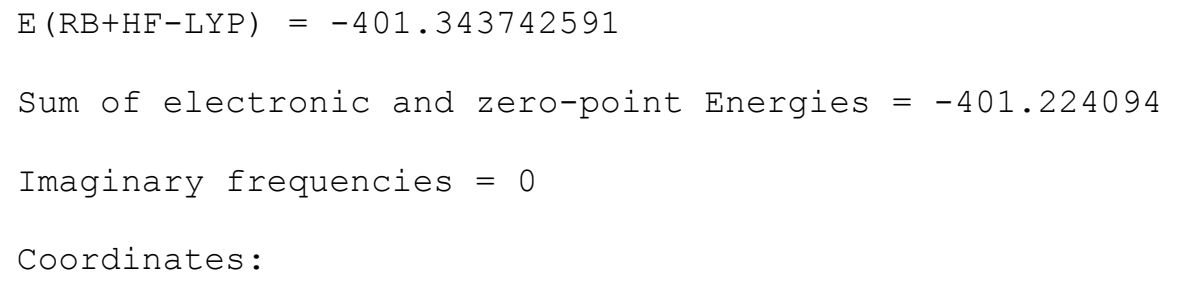

$\begin{array}{lrrr}\text { C } & -0.83521000 & 1.84809800 & 0.70539300 \\ \mathrm{C} & -0.83521000 & 1.84809800 & -0.70539300 \\ \mathrm{C} & -0.02551200 & 0.73807200 & -1.18748100 \\ \mathrm{C} & -0.02551200 & 0.73807200 & 1.18748100 \\ \mathrm{H} & -1.34370500 & 2.59265400 & 1.31122300 \\ \mathrm{H} & -1.34370500 & 2.59265400 & -1.31122300 \\ \mathrm{C} & -0.02551200 & -0.42452700 & -2.05041700 \\ \mathrm{H} & -0.29946500 & -0.48020400 & -3.09942700 \\ \mathrm{C} & -0.02551200 & -0.42452700 & 2.05041700 \\ \mathrm{H} & -0.29946500 & -0.48020400 & 3.09942700 \\ \mathrm{C} & 0.39475000 & -1.53706600 & 1.32427400 \\ \mathrm{H} & 0.59763100 & -2.54070200 & 1.67418400 \\ \mathrm{C} & 0.39475000 & -1.53706600 & -1.32427400 \\ \mathrm{H} & 0.59763100 & -2.54070200 & -1.67418400 \\ \mathrm{~N} & 0.71204000 & -1.13777100 & 0.00000000 \\ \mathrm{C} & 0.50077000 & 0.22099500 & 0.00000000\end{array}$

\section{Data for Structure 7 (planar conformer)}
$E(R B+H F-L Y P)=-401.333430371$
Sum of electronic and zero-point Energies $=-401.214932$
Imaginary frequencies $=1$
Coordinates:

$\begin{array}{lrrr}\text { C } & 0.00000000 & 2.11754200 & 0.41879900 \\ \mathrm{C} & 0.00000000 & 1.38180700 & 1.62451400 \\ \mathrm{C} & 0.00000000 & 1.19887400 & -0.70470700 \\ \mathrm{H} & 0.00000000 & 3.20226400 & 0.41014900 \\ \mathrm{H} & 0.00000000 & 1.73850400 & 2.64302800 \\ \mathrm{C} & 0.00000000 & -1.38180700 & 1.62451400 \\ \mathrm{H} & 0.00000000 & -1.73850400 & 2.64302800\end{array}$




$\begin{array}{lrrr}\text { C } & 0.00000000 & 0.71223200 & -2.08750200 \\ \mathrm{H} & 0.00000000 & 1.30728900 & -2.99464400 \\ \mathrm{C} & 0.00000000 & -0.71223200 & -2.08750200 \\ \mathrm{H} & 0.00000000 & -1.30728900 & -2.99464400 \\ \mathrm{C} & 0.00000000 & -2.11754200 & 0.41879900 \\ \mathrm{H} & 0.00000000 & -3.20226400 & 0.41014900 \\ \mathrm{C} & 0.00000000 & -1.19887400 & -0.70470700 \\ \mathrm{C} & 0.00000000 & 0.00000000 & -0.03908000 \\ \mathrm{~N} & 0.00000000 & 0.00000000 & 1.30059400\end{array}$

\section{Data for Structure 8 (bowl conformer)}

\begin{tabular}{|c|c|c|c|}
\hline \multicolumn{4}{|c|}{$E(R B+H F-L Y P)=-417.964459621$} \\
\hline Sum of electronic & and zero-point & Energies $=$ & 17.841733 \\
\hline \multicolumn{4}{|c|}{ Imaginary frequencies $=0$} \\
\hline \multicolumn{4}{|l|}{ Coordinates: } \\
\hline $\mathrm{C}$ & -2.01905900 & -0.70831300 & -0.09830700 \\
\hline $\mathrm{C}$ & -2.01918900 & 0.70795800 & -0.09825600 \\
\hline $\mathrm{C}$ & -0.69076600 & 1.16358500 & 0.14380300 \\
\hline $\mathrm{C}$ & -0.69055600 & -1.16372400 & 0.14373600 \\
\hline $\mathrm{H}$ & -2.88526700 & -1.32977200 & -0.26514600 \\
\hline $\mathrm{H}$ & -2.88551200 & 1.32926500 & -0.26506500 \\
\hline $\mathrm{C}$ & 0.39291100 & 2.06521100 & -0.12354000 \\
\hline $\mathrm{H}$ & 0.33251900 & 3.12493300 & -0.31399100 \\
\hline $\mathrm{C}$ & 0.39329300 & -2.06513100 & -0.12363900 \\
\hline $\mathrm{H}$ & 0.33311600 & -3.12485100 & -0.31416800 \\
\hline $\mathrm{C}$ & 1.57682200 & -1.33842800 & -0.12715200 \\
\hline $\mathrm{H}$ & 2.59230600 & -1.66916600 & -0.26538400 \\
\hline $\mathrm{C}$ & 1.57656600 & 1.33871800 & -0.12715500 \\
\hline $\mathrm{H}$ & 2.59199100 & 1.66962700 & -0.26540800 \\
\hline $\mathrm{N}$ & -0.03494000 & 0.00000000 & 0.41823600 \\
\hline $\mathrm{N}$ & 1.29218500 & 0.00011500 & 0.17493900 \\
\hline
\end{tabular}

\section{Data for Structure 8 (planar conformer)}

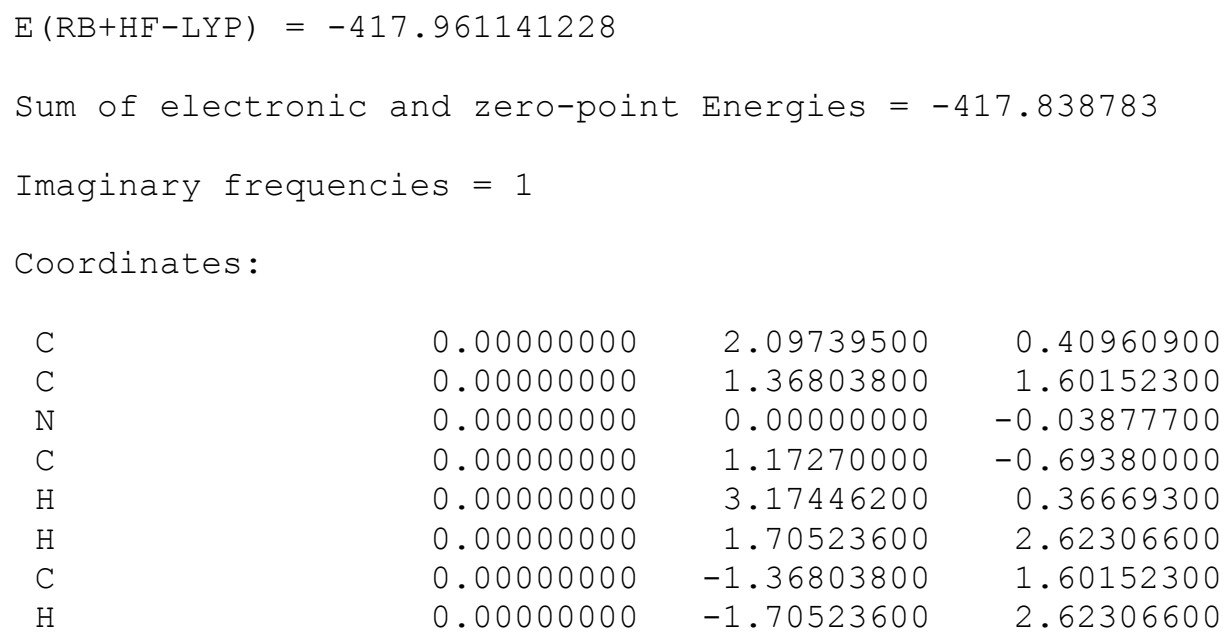




$\begin{array}{lrrr}\text { C } & 0.00000000 & 0.70926600 & -2.05378100 \\ \mathrm{H} & 0.00000000 & 1.32651000 & -2.93818800 \\ \mathrm{C} & 0.00000000 & -0.70926600 & -2.05378100 \\ \mathrm{H} & 0.00000000 & -1.32651000 & -2.93818800 \\ \mathrm{C} & 0.00000000 & -2.09739500 & 0.40960900 \\ \mathrm{H} & 0.00000000 & -3.17446200 & 0.36669300 \\ \mathrm{C} & 0.00000000 & -1.17270000 & -0.69380000 \\ \mathrm{~N} & 0.00000000 & 0.00000000 & 1.28652700\end{array}$

\section{Data for Structure 9 (bowl conformer)}

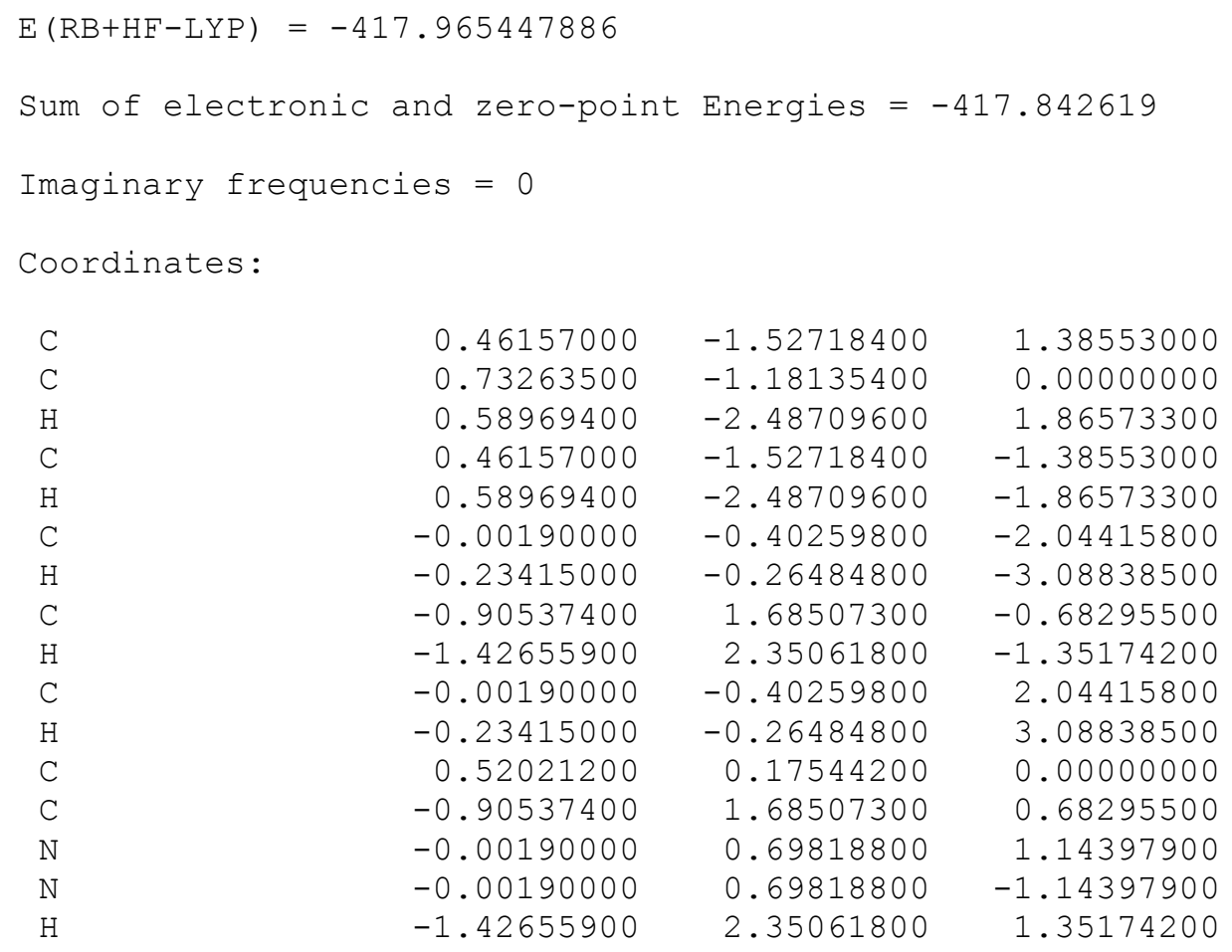

Data for Structure 9 (planar conformer)
$\mathrm{E}(\mathrm{RB}+\mathrm{HF}-\mathrm{LYP})=-417.953909785$
Sum of electronic and zero-point Energies $=-417.832097$
Imaginary frequencies $=1$
Coordinates:

$\begin{array}{lrrr}\text { C } & 0.00000000 & 0.69152400 & -1.98642300 \\ \mathrm{C} & 0.00000000 & -0.69152400 & -1.98642300 \\ \mathrm{H} & 0.00000000 & 1.35722600 & -2.83220300 \\ \mathrm{H} & 0.00000000 & -1.35722600 & -2.83220300 \\ \mathrm{C} & 0.00000000 & -2.11208400 & 0.40114500 \\ \mathrm{H} & 0.00000000 & -3.16750100 & 0.19027100 \\ \mathrm{C} & 0.00000000 & 2.11208400 & 0.40114500 \\ \mathrm{H} & 0.00000000 & 3.16750100 & 0.19027100 \\ \mathrm{C} & 0.00000000 & 1.44364400 & 1.62811300\end{array}$




$\begin{array}{lrrr}\mathrm{H} & 0.00000000 & 1.96717900 & 2.57312700 \\ \mathrm{C} & 0.00000000 & -1.44364400 & 1.62811300 \\ \mathrm{H} & 0.00000000 & -1.96717900 & 2.57312700 \\ \mathrm{C} & 0.00000000 & 0.00000000 & 1.39324500 \\ \mathrm{~N} & 0.00000000 & -1.13616600 & -0.64271400 \\ \mathrm{~N} & 0.00000000 & 1.13616600 & -0.64271400 \\ \mathrm{C} & 0.00000000 & 0.00000000 & 0.04368700\end{array}$

\section{Data for Structure 10 (bowl conformer)}

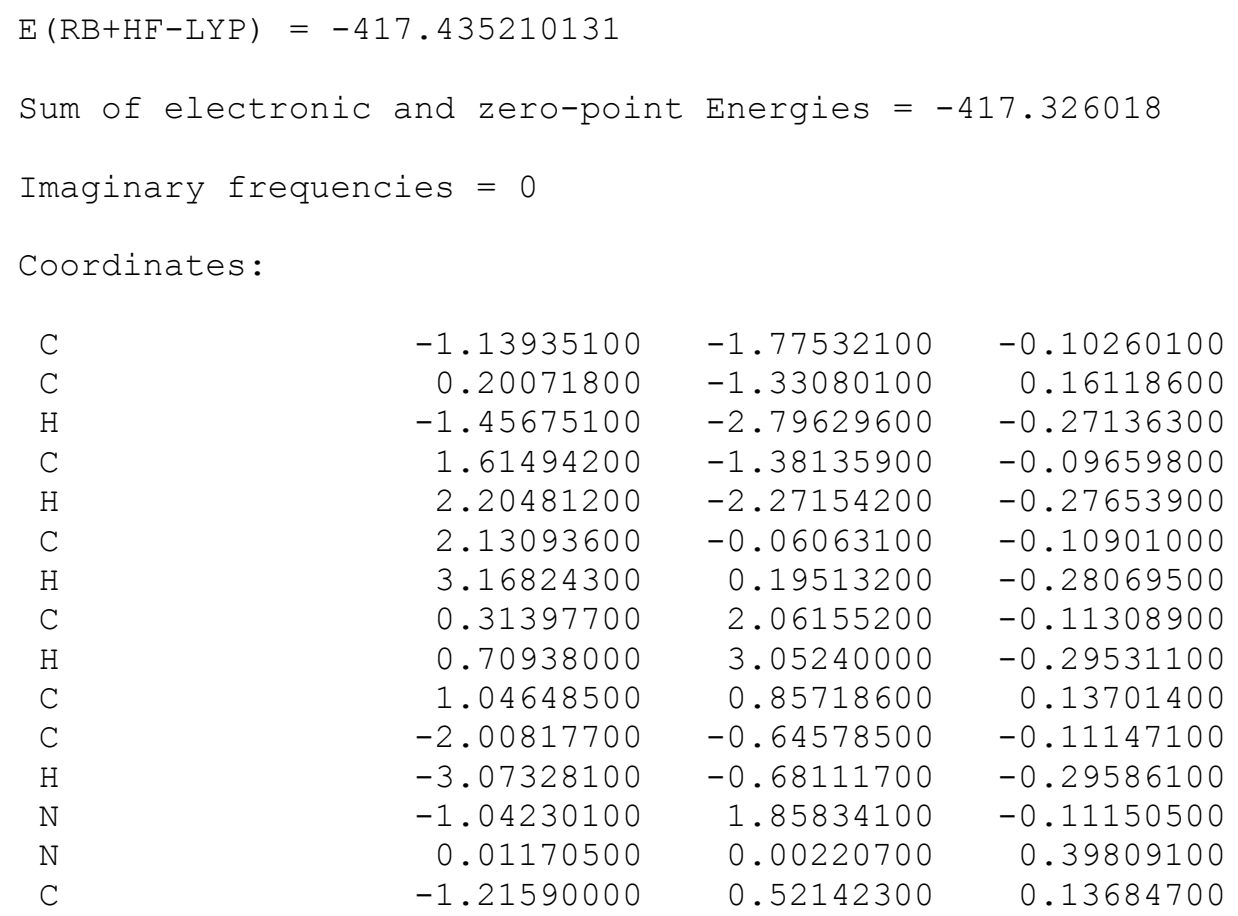

\section{Data for Structure 10 (planar conformer)}

$E(R B+H F-L Y P)=-417.432738270$

Sum of electronic and zero-point Energies $=-417.324032$

Imaginary frequencies $=1$

Coordinates:

$\begin{array}{lrrr}\text { C } & 2.10690100 & 0.20761600 & 0.00000000 \\ \text { C } & 0.46232100 & -1.24572900 & 0.00000000 \\ \text { N } & 0.00000000 & 0.01277000 & 0.00000000 \\ \text { C } & 0.91194800 & 1.00840500 & 0.00000000 \\ \text { H } & 3.13453400 & 0.54473200 & 0.00000000 \\ \text { C } & -0.76166000 & -2.00182800 & 0.00000000 \\ \text { H } & -0.86641500 & -3.07774000 & 0.00000000 \\ \mathrm{C} & 0.03984600 & 2.16403900 & 0.00000000 \\ \mathrm{H} & 0.33878300 & 3.20336400 & 0.00000000 \\ \mathrm{C} & -1.31140900 & 1.71167100 & 0.00000000 \\ \mathrm{H} & -2.17297000 & 2.36695200 & 0.00000000\end{array}$




$\begin{array}{lrrr}\mathrm{C} & -1.85282300 & -1.07418900 & 0.00000000 \\ \mathrm{H} & -2.89640600 & -1.35899100 & 0.00000000 \\ \mathrm{C} & -1.32449700 & 0.26798500 & 0.00000000 \\ \mathrm{~N} & 1.83410100 & -1.14221800 & 0.00000000\end{array}$

\section{Data for Structure 11 (bowl conformer)}

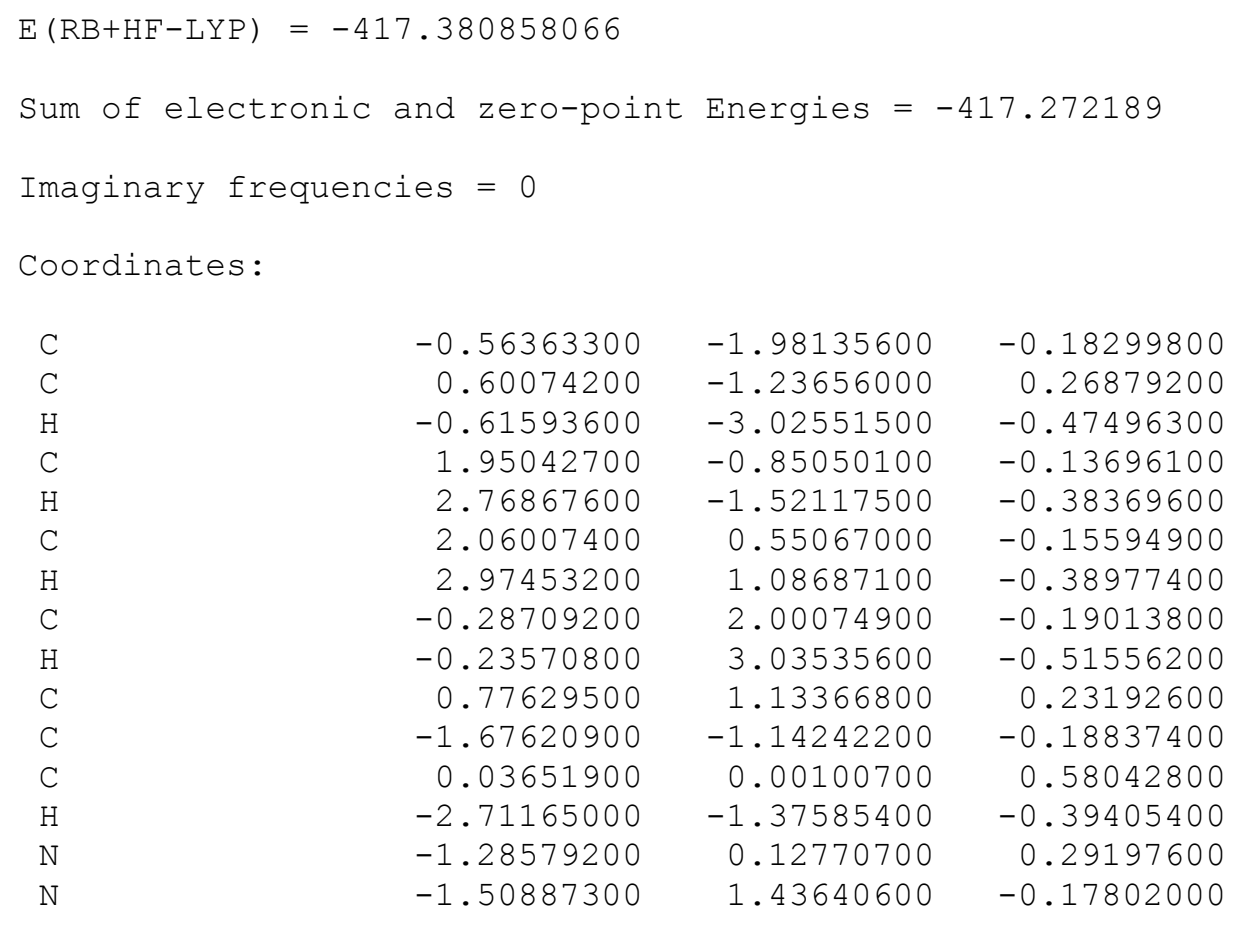

\section{Data for Structure 11 (planar conformer)}
$\mathrm{E}(\mathrm{RB}+\mathrm{HF}-\mathrm{LYP})=-417.368215076$
Sum of electronic and zero-point Energies = -417.260643
Imaginary frequencies $=1$
Coordinates:

$\begin{array}{lrrr}\mathrm{C} & -2.06069500 & -0.43204200 & 0.00000000 \\ \mathrm{C} & -1.19466700 & 0.71737600 & 0.00000000 \\ \mathrm{H} & -3.14349500 & -0.48736800 & 0.00000000 \\ \mathrm{C} & 1.30599800 & -1.66885600 & 0.00000000 \\ \mathrm{H} & 1.61587300 & -2.70165400 & 0.00000000 \\ \mathrm{C} & -0.67930500 & 2.09944600 & 0.00000000 \\ \mathrm{H} & -1.25638500 & 3.01707300 & 0.00000000 \\ \mathrm{C} & 0.74120100 & 2.06812700 & 0.00000000 \\ \mathrm{H} & 1.34954100 & 2.96705300 & 0.00000000 \\ \mathrm{C} & 2.08934200 & -0.48985700 & 0.00000000 \\ \mathrm{H} & 3.17313800 & -0.52326500 & 0.00000000 \\ \mathrm{C} & 1.21236200 & 0.67451900 & 0.00000000 \\ \mathrm{~N} & -0.05205200 & -1.27213800 & 0.00000000 \\ \mathrm{~N} & -1.40853300 & -1.63654000 & 0.00000000\end{array}$



C
0.00000000
0.04610600
0.00000000

\section{Data for Structure 12 (bowl conformer)}

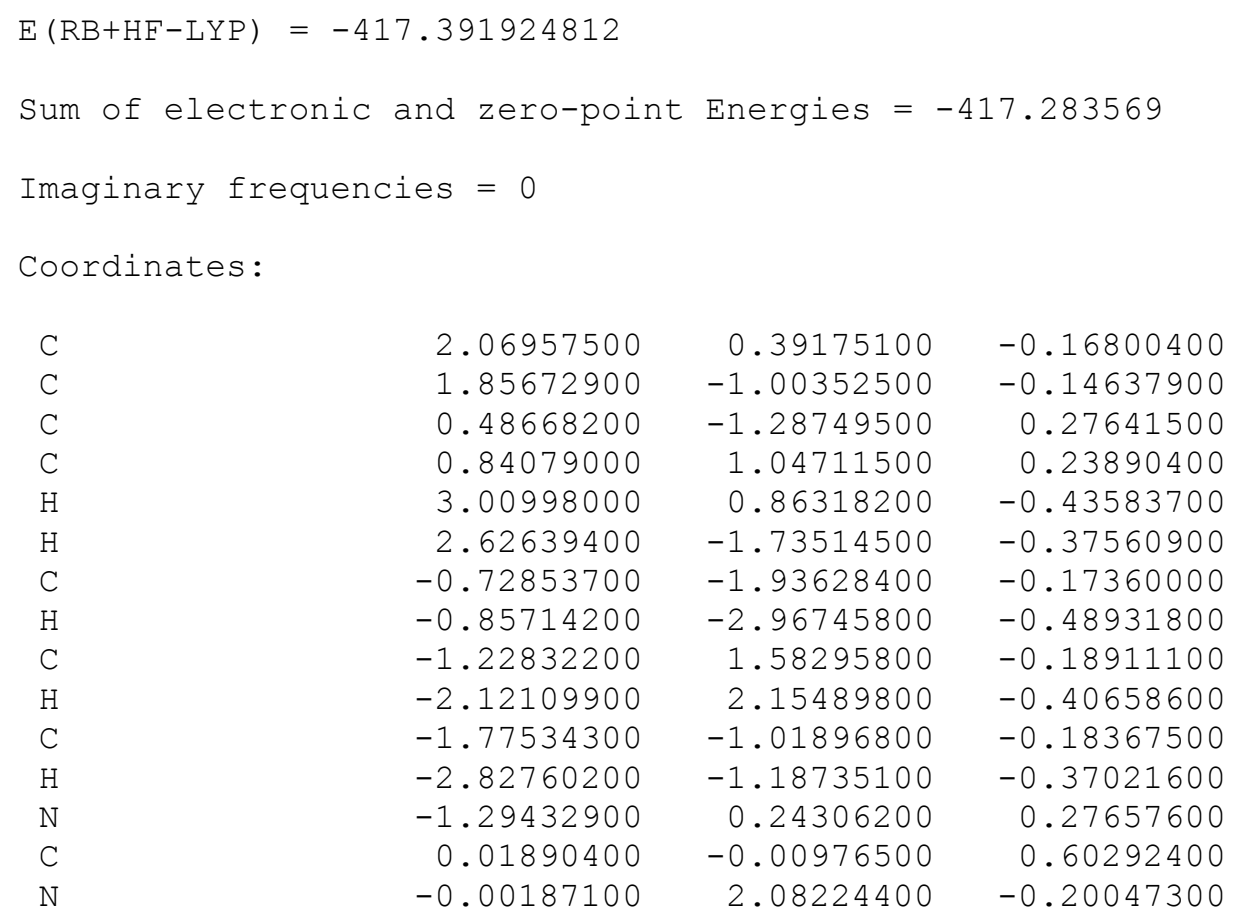

\section{Data for Structure 12 (planar conformer)}

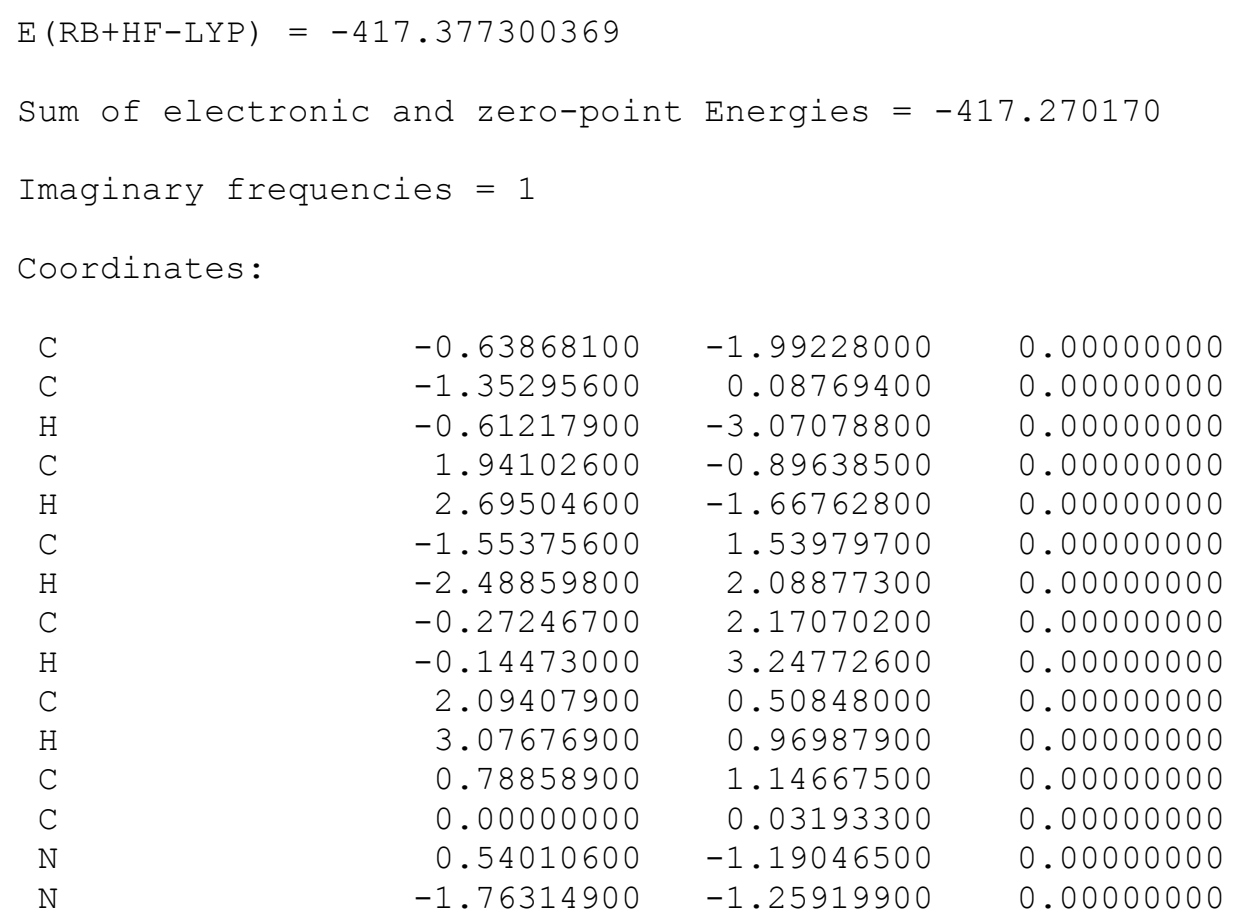


Data for Structure 13 (bowl conformer)

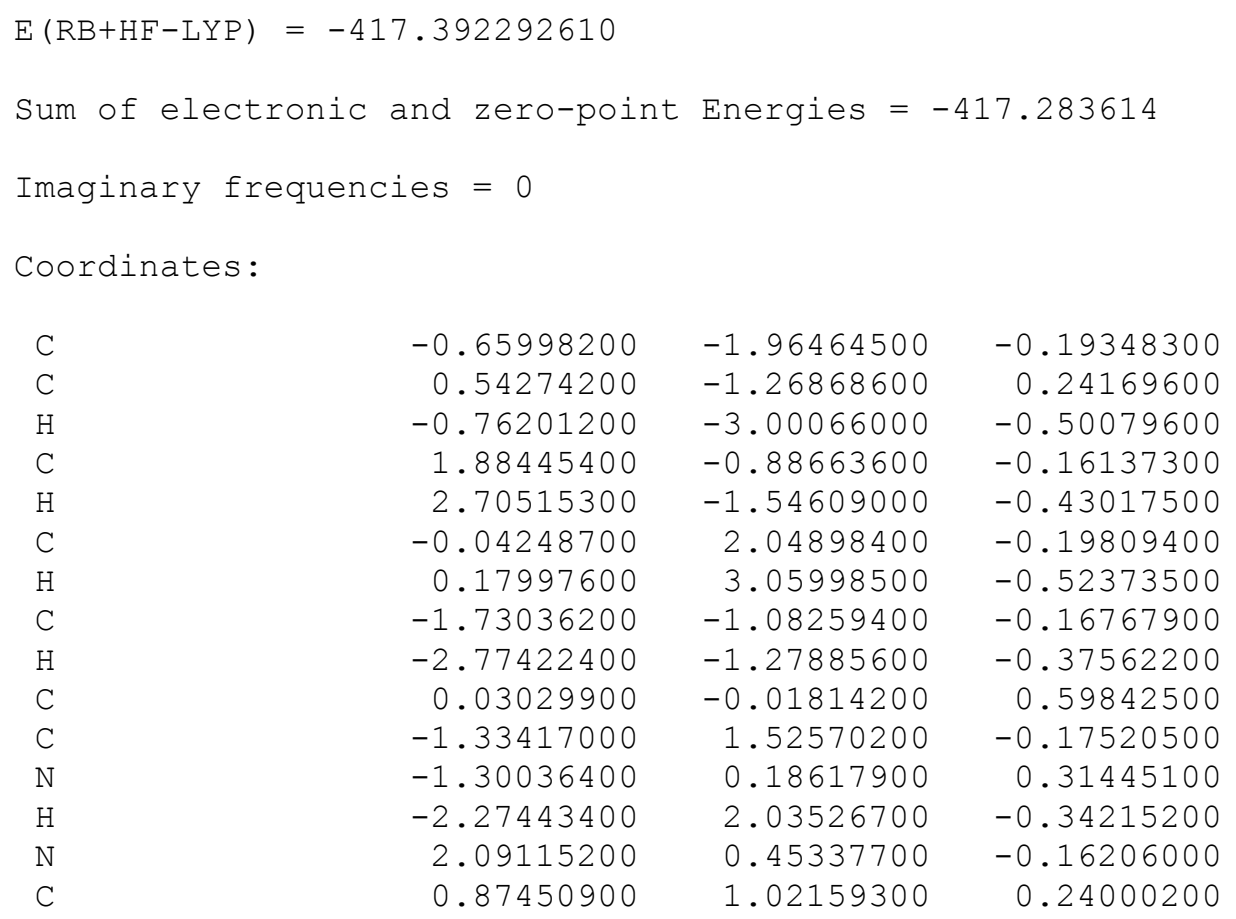

Data for Structure 13 (planar conformer)

$\begin{array}{llll}\mathrm{E}(\mathrm{RB}+\mathrm{HF}-\mathrm{LYP})=-417.377859043 & \\ \text { Sum of electronic and zero-point Energies }=-417.270326 \\ \text { Imaginary frequencies }=1 & & & \\ & & & \\ \text { Coordinates: } & & & \\ & & & \\ \mathrm{C} & -1.77905800 & -1.18758700 & 0.00000000 \\ \mathrm{C} & -0.63209500 & -2.01914200 & 0.00000000 \\ \mathrm{C} & -1.34471000 & 0.19720300 & 0.00000000 \\ \mathrm{H} & -2.78265800 & -1.59848600 & 0.00000000 \\ \mathrm{H} & -0.57039400 & -3.09622600 & 0.00000000 \\ \mathrm{C} & 1.92460300 & -0.90970400 & 0.00000000 \\ \mathrm{H} & 2.67035900 & -1.69077500 & 0.00000000 \\ \mathrm{C} & -0.27606000 & 2.14180500 & 0.00000000 \\ \mathrm{H} & -0.18346800 & 3.22255500 & 0.00000000 \\ \mathrm{C} & 2.10692800 & 0.48693600 & 0.00000000 \\ \mathrm{H} & 3.09523900 & 0.93342100 & 0.00000000 \\ \mathrm{C} & 0.80057900 & 1.14506100 & 0.00000000 \\ \mathrm{C} & 0.00000000 & 0.03751000 & 0.00000000 \\ \mathrm{~N} & 0.52829900 & -1.18741400 & 0.00000000 \\ \mathrm{~N} & -1.53261300 & 1.59841600 & 0.00000000\end{array}$

Data for Structure 14 (bowl conformer)

$E(R B+H F-L Y P)=-433.988700883$ 
Sum of electronic and zero-point Energies $=-433.877552$

Imaginary frequencies $=0$

Coordinates:

$\begin{array}{lrrr}\text { C } & -0.67681700 & -1.96155700 & -0.13796200 \\ \mathrm{C} & 0.52587400 & -1.23790800 & 0.17277800 \\ \mathrm{H} & -0.77046200 & -3.01358100 & -0.35544100 \\ \mathrm{C} & 1.90135900 & -0.95462000 & -0.10684700 \\ \mathrm{H} & 2.67415700 & -1.68220400 & -0.30237600 \\ \mathrm{C} & 2.08355000 & 0.44477100 & -0.12232200 \\ \mathrm{H} & 3.01705700 & 0.94921000 & -0.31744500 \\ \mathrm{C} & -0.18551500 & 2.03376200 & -0.14777300 \\ \mathrm{H} & -0.07325400 & 3.07968500 & -0.38719300 \\ \mathrm{C} & 0.82561500 & 1.06817100 & 0.15171200 \\ \mathrm{C} & -1.73981100 & -1.06440200 & -0.14908700 \\ \mathrm{H} & -2.78944000 & -1.22518500 & -0.32657400 \\ \mathrm{~N} & -1.25825300 & 0.19501900 & 0.20831100 \\ \mathrm{~N} & -1.42137800 & 1.51052100 & -0.14283500 \\ \mathrm{~N} & 0.04197500 & -0.00228700 & 0.46681500\end{array}$

\section{Data for Structure 14 (planar conformer)}

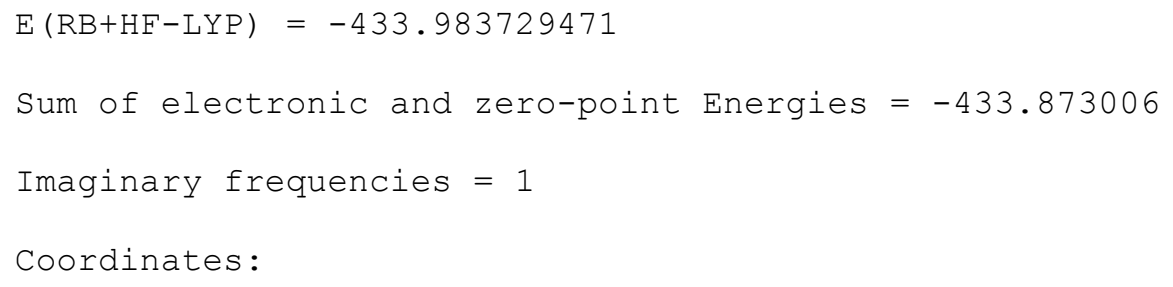

$\begin{array}{lrrr}\mathrm{C} & -2.04627900 & -0.42212400 & 0.00000000 \\ \mathrm{~N} & 0.00000000 & 0.04609200 & 0.00000000 \\ \mathrm{C} & -1.16503100 & 0.71015300 & 0.00000000 \\ \mathrm{H} & -3.12393500 & -0.44911800 & 0.00000000 \\ \mathrm{C} & 1.28561500 & -1.65102200 & 0.00000000 \\ \mathrm{H} & 1.57124900 & -2.68770300 & 0.00000000 \\ \mathrm{C} & -0.67026000 & 2.06860000 & 0.00000000 \\ \mathrm{H} & -1.26820800 & 2.96575800 & 0.00000000 \\ \mathrm{C} & 0.74315900 & 2.03701400 & 0.00000000 \\ \mathrm{H} & 1.37703300 & 2.90989400 & 0.00000000 \\ \mathrm{C} & 2.06560300 & -0.48547800 & 0.00000000 \\ \mathrm{H} & 3.14333700 & -0.48825800 & 0.00000000 \\ \mathrm{C} & 1.18569400 & 0.65904700 & 0.00000000 \\ \mathrm{~N} & -0.05668200 & -1.26251600 & 0.00000000 \\ \mathrm{~N} & -1.38481600 & -1.60467900 & 0.00000000\end{array}$

\section{Data for Structure 15 (bowl conformer)}

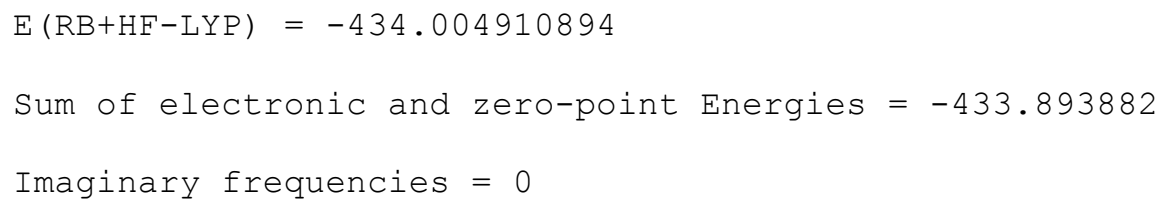


Coordinates :

$\begin{array}{lrrr}\text { C } & 2.09525200 & 0.23164000 & -0.13155400 \\ \text { C } & 1.76679300 & -1.14871000 & -0.11621700 \\ \text { C } & 0.37912700 & -1.29239100 & 0.18098500 \\ \text { C } & 0.91179600 & 0.96094400 & 0.15460800 \\ \text { H } & 3.06821400 & 0.64279200 & -0.34943300 \\ H & 2.46102300 & -1.95121600 & -0.31300400 \\ \mathrm{C} & -0.89129100 & -1.88611300 & -0.13173400 \\ \mathrm{H} & -1.09131100 & -2.92049200 & -0.36460900 \\ \mathrm{C} & -1.09577700 & 1.69433100 & -0.15347500 \\ \mathrm{H} & -1.94559800 & 2.33327000 & -0.32999800 \\ \mathrm{C} & -1.85588600 & -0.88976700 & -0.14467100 \\ \mathrm{H} & -2.91797600 & -0.95540900 & -0.31029000 \\ \mathrm{~N} & -1.25193500 & 0.33808300 & 0.19315800 \\ \mathrm{~N} & 0.16864000 & 2.07712800 & -0.15178100 \\ \mathrm{~N} & 0.02123200 & -0.01071700 & 0.49000700\end{array}$

\section{Data for Structure 15 (planar conformer)}

$\mathrm{E}(\mathrm{RB}+\mathrm{HF}-\mathrm{LYP})=-433.998746659$

Sum of electronic and zero-point Energies $=-433.888194$

Imaginary frequencies $=1$

Coordinates:

$\begin{array}{lrrr}\mathrm{C} & -0.83363900 & -1.89623200 & 0.00000000 \\ \mathrm{~N} & 0.00000000 & 0.02962900 & 0.00000000 \\ \mathrm{C} & -1.31738800 & 0.22463300 & 0.00000000 \\ \mathrm{H} & -0.90211700 & -2.97009500 & 0.00000000 \\ \mathrm{C} & 1.82055700 & -1.07042400 & 0.00000000 \\ \mathrm{H} & 2.47502400 & -1.92380600 & 0.00000000 \\ \mathrm{C} & -1.37574800 & 1.65967400 & 0.00000000 \\ \mathrm{H} & -2.26345400 & 2.27109000 & 0.00000000 \\ \mathrm{C} & -0.04264400 & 2.15313600 & 0.00000000 \\ \mathrm{H} & 0.22412100 & 3.19798900 & 0.00000000 \\ \mathrm{C} & 2.11370100 & 0.29559000 & 0.00000000 \\ \mathrm{H} & 3.11847400 & 0.68775100 & 0.00000000 \\ \mathrm{C} & 0.87606400 & 1.03965600 & 0.00000000 \\ \mathrm{~N} & 0.41361800 & -1.22556800 & 0.00000000 \\ \mathrm{~N} & -1.85611400 & -1.04679200 & 0.00000000\end{array}$

\section{Data for Structure 16 (bowl conformer)}

$E(R B+H F-L Y P)=-434.004577304$

Sum of electronic and zero-point Energies $=-433.893314$

Imaginary frequencies $=0$

Coordinates:

C

$1.72949500-1.16790900-0.12896500$ 


$\begin{array}{lrrr}\text { C } & 0.34257200 & -1.31195100 & 0.15606500 \\ \mathrm{C} & 1.00638300 & 0.86262600 & 0.15227600 \\ \mathrm{H} & 2.44347300 & -1.94870700 & -0.34145400 \\ \mathrm{C} & -0.95716500 & -1.86008100 & -0.14604500 \\ \mathrm{H} & -1.20088400 & -2.88657700 & -0.36958500 \\ \mathrm{C} & 0.26614900 & 2.05480800 & -0.14887300 \\ \mathrm{H} & 0.65603600 & 3.03234200 & -0.38247000 \\ \mathrm{C} & -1.08187600 & 1.72404800 & -0.13892600 \\ \mathrm{H} & -1.94537600 & 2.34883400 & -0.29597100 \\ \mathrm{C} & -1.87756900 & -0.82776400 & -0.13380700 \\ \mathrm{H} & -2.94175400 & -0.85053700 & -0.30094700 \\ \mathrm{~N} & 0.03199000 & -0.02180900 & 0.48041700 \\ \mathrm{~N} & -1.23268700 & 0.37383100 & 0.22304100 \\ \mathrm{~N} & 2.11792100 & 0.14254700 & -0.12916100\end{array}$

\section{Data for Structure 16 (planar conformer)}

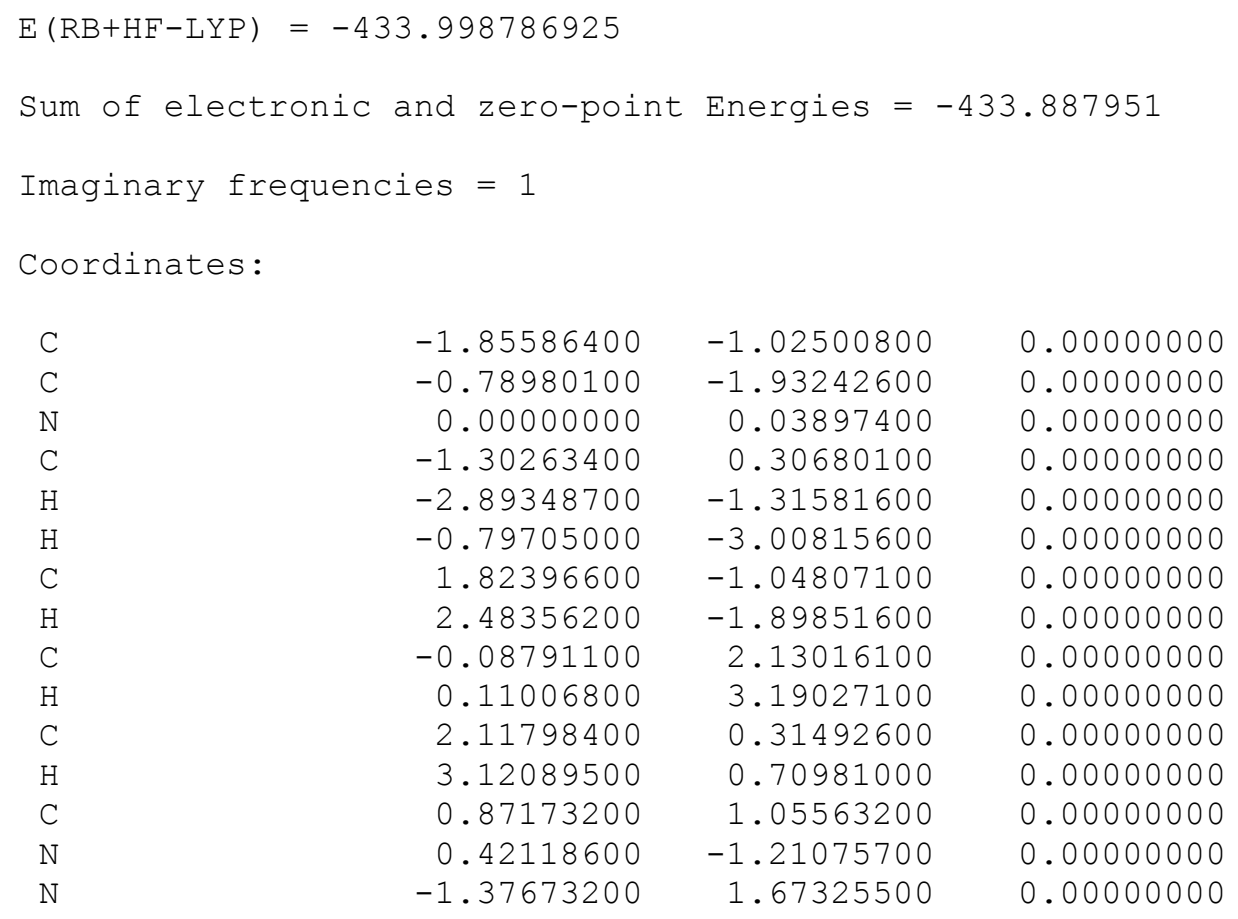

\section{Data for Structure 17 (bowl conformer)}
$E(R B+H F-L Y P)=-433.461774449$
Sum of electronic and zero-point Energies $=-433.364442$
Imaginary frequencies $=0$
Coordinates:

$\begin{array}{lrrr}\mathrm{C} & 0.29568900 & -1.54276700 & 1.38615500 \\ \mathrm{C} & 0.53939500 & -1.24247800 & 0.00000000 \\ \mathrm{H} & 0.33633500 & -2.52268400 & 1.84548300 \\ \mathrm{C} & 0.29568900 & -1.54276700 & -1.38615500\end{array}$




$\begin{array}{lrrr}\mathrm{H} & 0.33633500 & -2.52268400 & -1.84548300 \\ \mathrm{C} & -0.04219500 & -0.35029500 & 2.07486800 \\ \mathrm{H} & -0.28337800 & -0.28229400 & 3.12632600 \\ \mathrm{C} & -0.04219500 & 0.72352500 & -1.11265300 \\ \mathrm{C} & -0.04219500 & 0.72352500 & 1.11265300 \\ \mathrm{C} & -0.04219500 & -0.35029500 & -2.07486800 \\ \mathrm{H} & -0.28337800 & -0.28229400 & -3.12632600 \\ \mathrm{~N} & -0.64302700 & 1.87374200 & 0.69014300 \\ \mathrm{~N} & -0.64302700 & 1.87374200 & -0.69014300 \\ \mathrm{~N} & 0.44635900 & 0.12384000 & 0.00000000\end{array}$

\section{Data for Structure 17 (planar conformer)}

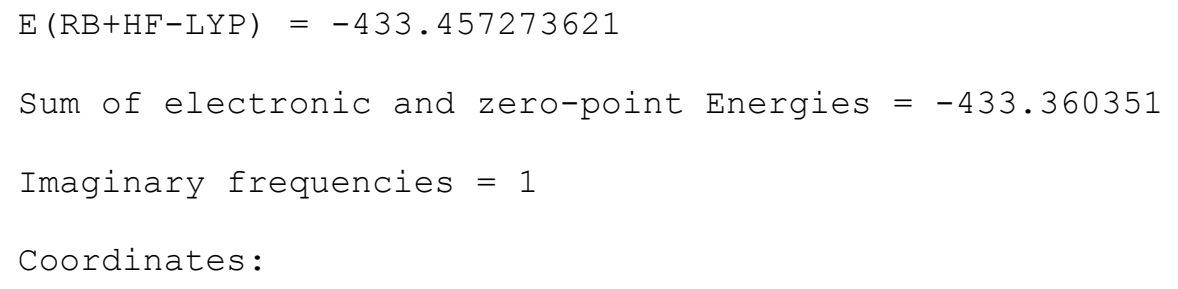

$\begin{array}{lrrr}\mathrm{C} & 0.02322200 & 1.31229600 & 0.00000000 \\ \mathrm{~N} & -0.03622400 & -0.01870500 & 0.00000000 \\ \mathrm{C} & 1.14655700 & -0.63239800 & 0.00000000 \\ \mathrm{C} & -1.38900700 & 1.64576800 & 0.00000000 \\ \mathrm{H} & -1.83039900 & 2.63181100 & 0.00000000 \\ \mathrm{C} & 0.72948000 & -2.02219200 & 0.00000000 \\ \mathrm{H} & 1.36305200 & -2.89727200 & 0.00000000 \\ \mathrm{C} & -0.69727600 & -2.04789400 & 0.00000000 \\ \mathrm{H} & -1.28693100 & -2.95544800 & 0.00000000 \\ \mathrm{C} & -2.12420100 & 0.42274000 & 0.00000000 \\ \mathrm{H} & -3.20497900 & 0.36556200 & 0.00000000 \\ \mathrm{C} & -1.19992900 & -0.69084500 & 0.00000000 \\ \mathrm{~N} & 1.36850200 & 1.59294800 & 0.00000000 \\ \mathrm{~N} & 2.06178800 & 0.39280100 & 0.00000000\end{array}$

\section{Data for Structure 18 (bowl conformer)}
$E(R B+H F-L Y P)=-433.483726155$
Sum of electronic and zero-point Energies $=-433.385672$
Imaginary frequencies $=0$
Coordinates:

$\begin{array}{lrrr}\text { C } & 1.30022700 & 1.58794700 & -0.14019500 \\ \mathrm{C} & 1.30932300 & 0.18811900 & 0.15005900 \\ \mathrm{C} & -0.79877300 & 1.04473800 & 0.17643500 \\ \mathrm{H} & 2.13962000 & 2.22925100 & -0.37129900 \\ \mathrm{C} & -1.84662800 & -0.99687400 & -0.12377400 \\ \mathrm{H} & -2.61650500 & -1.73069700 & -0.32373100 \\ \mathrm{C} & 0.78523600 & -1.91351000 & -0.12417400 \\ \mathrm{H} & 0.94685400 & -2.96055000 & -0.34847000 \\ \mathrm{C} & -0.46039600 & -1.26601400 & 0.17779000\end{array}$




$\begin{array}{lrrr}\mathrm{N} & 0.02489700 & 2.09855400 & -0.12818800 \\ \mathrm{~N} & 1.84319600 & -1.05042100 & -0.13705100 \\ \mathrm{C} & -2.05201400 & 0.40723000 & -0.12039700 \\ \mathrm{H} & -2.99001200 & 0.89617600 & -0.34808500 \\ \mathrm{~N} & 0.00307600 & -0.01156100 & 0.46768400\end{array}$

\section{Data for Structure 18 (planar conformer)}

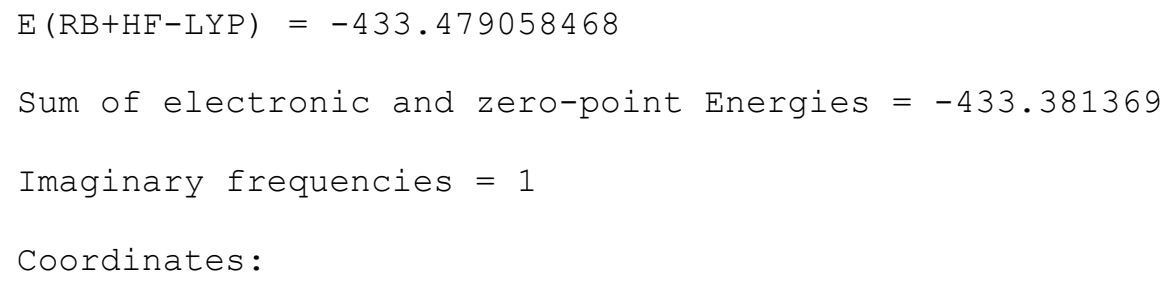

$\begin{array}{lrrr}\mathrm{C} & -1.22055500 & -1.70670700 & 0.00000000 \\ \mathrm{C} & 0.86715900 & -0.99935400 & 0.00000000 \\ \mathrm{~N} & 0.00000000 & 0.01317300 & 0.00000000 \\ \mathrm{C} & -1.30520600 & -0.26902200 & 0.00000000 \\ \mathrm{H} & -2.02344900 & -2.42989200 & 0.00000000 \\ \mathrm{C} & 2.11865300 & -0.27629100 & 0.00000000 \\ \mathrm{H} & 3.11642300 & -0.69364000 & 0.00000000 \\ \mathrm{C} & -0.93090500 & 1.89642500 & 0.00000000 \\ \mathrm{H} & -1.18414900 & 2.94847900 & 0.00000000 \\ \mathrm{C} & 1.82761400 & 1.12291100 & 0.00000000 \\ \mathrm{H} & 2.57761300 & 1.90173700 & 0.00000000 \\ \mathrm{C} & 0.38561200 & 1.30072500 & 0.00000000 \\ \mathrm{~N} & 0.09078900 & -2.14024400 & 0.00000000 \\ \mathrm{~N} & -1.93945500 & 0.96438400 & 0.00000000\end{array}$

\section{Data for Structure 19 (bowl conformer)}
$\mathrm{E}(\mathrm{RB}+\mathrm{HF}-\mathrm{LYP})=-433.483463065$
Sum of electronic and zero-point Energies $=-433.385490$
Imaginary frequencies $=0$
Coordinates:

$\begin{array}{lrrr}\mathrm{C} & -0.47746000 & 1.46030400 & 1.39853000 \\ \mathrm{C} & -0.15211300 & 1.35400500 & 0.00000000 \\ \mathrm{C} & 0.31226600 & -0.56605700 & 1.16012100 \\ \mathrm{H} & -0.89796100 & 2.31127900 & 1.91813300 \\ \mathrm{C} & -0.47746000 & 1.46030400 & -1.39853000 \\ \mathrm{H} & -0.89796100 & 2.31127900 & -1.91813300 \\ \mathrm{C} & 0.31226600 & -1.92789800 & -0.71318800 \\ \mathrm{H} & 0.28957100 & -2.81320900 & -1.33414400 \\ \mathrm{C} & 0.31226600 & -0.56605700 & -1.16012100 \\ \mathrm{~N} & -0.20268400 & 0.31310700 & 2.08380200 \\ \mathrm{C} & 0.31226600 & -1.92789800 & 0.71318800 \\ \mathrm{H} & 0.28957100 & -2.81320900 & 1.33414400 \\ \mathrm{~N} & -0.20268400 & 0.31310700 & -2.08380200 \\ \mathrm{~N} & 0.45745300 & 0.12859100 & 0.00000000\end{array}$




\section{Data for Structure 19 (planar conformer)}

\begin{tabular}{|c|c|c|c|}
\hline \multicolumn{4}{|c|}{$E(R B+H F-L Y P)=-433.478318958$} \\
\hline Sum of electronic & and zero-point & Energies $=$ & -433.380704 \\
\hline \multicolumn{4}{|c|}{ Imaginary frequencies $=1$} \\
\hline \multicolumn{4}{|l|}{ Coordinates: } \\
\hline $\mathrm{C}$ & 2.07825200 & 0.46240700 & 0.00000000 \\
\hline $\mathrm{C}$ & 0.05148800 & 1.31975600 & 0.00000000 \\
\hline $\mathrm{N}$ & 0.02716000 & -0.01421600 & 0.00000000 \\
\hline $\mathrm{C}$ & 1.19195800 & -0.68603800 & 0.00000000 \\
\hline $\mathrm{H}$ & 3.15929200 & 0.46920800 & 0.00000000 \\
\hline $\mathrm{C}$ & -1.36107400 & 1.61551800 & 0.00000000 \\
\hline $\mathrm{H}$ & -1.82643600 & 2.59106300 & 0.00000000 \\
\hline $\mathrm{C}$ & 0.64264700 & -2.02793300 & 0.00000000 \\
\hline $\mathrm{H}$ & 1.17794100 & -2.96713500 & 0.00000000 \\
\hline $\mathrm{C}$ & -2.07876300 & 0.37081700 & 0.00000000 \\
\hline $\mathrm{H}$ & -3.15620700 & 0.28491700 & 0.00000000 \\
\hline $\mathrm{C}$ & -1.11509900 & -0.70372300 & 0.00000000 \\
\hline $\mathrm{N}$ & -0.72974600 & -2.03130400 & 0.00000000 \\
\hline $\mathrm{N}$ & 1.39372300 & 1.65124300 & 0.00000000 \\
\hline
\end{tabular}

\section{Data for Structure 20 (bowl conformer)}

$E(R B+H F-L Y P)=-433.485167798$

Sum of electronic and zero-point Energies $=-433.387074$

Imaginary frequencies $=0$

Coordinates:

$\begin{array}{lrrr}\text { C } & -0.00086500 & -0.49519500 & 1.99513100 \\ \mathrm{C} & -0.00086500 & 0.68007800 & 1.17497000 \\ \mathrm{C} & 0.47980000 & -1.21133000 & 0.00000000 \\ \mathrm{H} & -0.20290500 & -0.56116500 & 3.05623500 \\ \mathrm{C} & -0.63422800 & 1.89207700 & 0.70620400 \\ \mathrm{H} & -1.06657100 & 2.67239600 & 1.31958100 \\ \mathrm{C} & -0.00086500 & -0.49519500 & -1.99513100 \\ \mathrm{H} & -0.20290500 & -0.56116500 & -3.05623500 \\ \mathrm{C} & -0.63422800 & 1.89207700 & -0.70620400 \\ \mathrm{H} & -1.06657100 & 2.67239600 & -1.31958100 \\ \mathrm{C} & -0.00086500 & 0.68007800 & -1.17497000 \\ \mathrm{~N} & 0.29644300 & -1.63302600 & -1.28644400 \\ \mathrm{~N} & 0.29644300 & -1.63302600 & 1.28644400 \\ \mathrm{~N} & 0.44877900 & 0.14062500 & 0.00000000\end{array}$

\section{Data for Structure 20 (planar conformer)}

$E(R B+H F-L Y P)=-433.480425752$

Sum of electronic and zero-point Energies $=-433.382671$ 
Imaginary frequencies $=1$

Coordinates:

$\begin{array}{lrrr}\mathrm{C} & 2.03592100 & 0.45463300 & 0.00000000 \\ \mathrm{C} & 0.00000000 & 1.25551600 & 0.00000000 \\ \mathrm{~N} & 0.00000000 & -0.07184500 & 0.00000000 \\ \mathrm{C} & 1.18245500 & -0.71035300 & 0.00000000 \\ \mathrm{H} & 3.11646800 & 0.48829100 & 0.00000000 \\ \mathrm{C} & 0.71102900 & -2.08571000 & 0.00000000 \\ \mathrm{H} & 1.31931700 & -2.98046200 & 0.00000000 \\ \mathrm{C} & -0.71102900 & -2.08571000 & 0.00000000 \\ \mathrm{H} & -1.31931700 & -2.98046200 & 0.00000000 \\ \mathrm{C} & -2.03592100 & 0.45463300 & 0.00000000 \\ \mathrm{H} & -3.11646800 & 0.48829100 & 0.00000000 \\ \mathrm{C} & -1.18245500 & -0.71035300 & 0.00000000 \\ \mathrm{~N} & -1.32073100 & 1.63551400 & 0.00000000 \\ \mathrm{~N} & 1.32073100 & 1.63551400 & 0.00000000\end{array}$

\title{
Data for Structure 21 (bowl conformer)
}

$\mathrm{E}(\mathrm{RB}+\mathrm{HF}-\mathrm{LYP})=-434.351255284$

Sum of electronic and zero-point Energies = -434.227341

Imaginary frequencies $=0$

Coordinates:

$\begin{array}{lrrr}\mathrm{C} & 0.36588200 & -1.56039300 & 1.38089200 \\ \mathrm{C} & -0.01404800 & -0.39829200 & 2.03707900 \\ \mathrm{~N} & 0.47673500 & 0.11272400 & 0.00000000 \\ \mathrm{C} & 0.55094600 & -1.23844700 & 0.00000000 \\ \mathrm{H} & 0.45821500 & -2.52866700 & 1.84535000 \\ \mathrm{H} & -0.24323000 & -0.23230700 & 3.07722200 \\ \mathrm{C} & -0.73305300 & 1.78372200 & 0.68249300 \\ \mathrm{H} & -1.15074600 & 2.50372800 & 1.36596500 \\ \mathrm{C} & 0.36588200 & -1.56039300 & -1.38089200 \\ \mathrm{H} & 0.45821500 & -2.52866700 & -1.84535000 \\ \mathrm{C} & -0.01404800 & -0.39829200 & -2.03707900 \\ \mathrm{H} & -0.24323000 & -0.23230700 & -3.07722200 \\ \mathrm{C} & -0.73305300 & 1.78372200 & -0.68249300 \\ \mathrm{H} & -1.15074600 & 2.50372800 & -1.36596500 \\ \mathrm{~N} & -0.01404800 & 0.66111900 & -1.13136400 \\ \mathrm{~N} & -0.01404800 & 0.66111900 & 1.13136400\end{array}$

\section{Data for Structure 21 (planar conformer)}

\author{
$E(R B+H F-L Y P)=-434.344468030$ \\ Sum of electronic and zero-point Energies $=-434.221133$ \\ Imaginary frequencies $=1$ \\ Coordinates:
}




$\begin{array}{lrrr}\text { C } & 0.00000000 & 0.68740600 & -1.97440700 \\ \mathrm{C} & 0.00000000 & -0.68740600 & -1.97440700 \\ \mathrm{~N} & 0.00000000 & 0.00000000 & 0.04239500 \\ \mathrm{H} & 0.00000000 & 1.37037200 & -2.80602900 \\ \mathrm{H} & 0.00000000 & -1.37037200 & -2.80602900 \\ \mathrm{C} & 0.0000000 & -2.08626800 & 0.39569500 \\ \mathrm{H} & 0.00000000 & -3.13927200 & 0.17151900 \\ \mathrm{C} & 0.00000000 & 2.08626800 & 0.39569500 \\ \mathrm{H} & 0.00000000 & 3.13927200 & 0.17151900 \\ \mathrm{C} & 0.00000000 & 1.42265600 & 1.61986000 \\ \mathrm{H} & 0.00000000 & 1.91592700 & 2.57777600 \\ \mathrm{C} & 0.00000000 & -1.42265600 & 1.61986000 \\ \mathrm{H} & 0.0000000 & -1.91592700 & 2.57777600 \\ \mathrm{C} & 0.00000000 & 0.00000000 & 1.36661200 \\ \mathrm{~N} & 0.00000000 & -1.12893600 & -0.63405200 \\ \mathrm{~N} & 0.00000000 & 1.12893600 & -0.63405200\end{array}$

\section{Data for Structure 22 (bowl conformer)}

\begin{tabular}{|c|c|c|c|}
\hline \multicolumn{4}{|c|}{ Sum of electronic and zero-po } \\
\hline \multicolumn{4}{|c|}{ Imaginary frequencies $=0$} \\
\hline \multicolumn{4}{|c|}{ Coordinates: } \\
\hline C & -0.68266100 & 1.94448700 & -0.16264900 \\
\hline $\mathrm{C}$ & 0.68266100 & 1.94448700 & -0.16264900 \\
\hline $\mathrm{N}$ & 1.13064900 & 0.65278100 & 0.23412300 \\
\hline $\mathrm{C}$ & 0.00000000 & 0.00000000 & 0.50691000 \\
\hline $\mathrm{N}$ & -1.13064900 & 0.65278100 & 0.23412300 \\
\hline $\mathrm{H}$ & -1.36320900 & 2.75831500 & -0.35044400 \\
\hline $\mathrm{H}$ & 1.36320900 & 2.75831500 & -0.35044400 \\
\hline $\mathrm{C}$ & 2.02530600 & -0.38104200 & -0.16264900 \\
\hline $\mathrm{H}$ & 3.07037600 & -0.19858400 & -0.35044400 \\
\hline C & -2.02530600 & -0.38104200 & -0.16264900 \\
\hline $\mathrm{H}$ & -3.07037600 & -0.19858400 & -0.35044400 \\
\hline $\mathrm{C}$ & -1.34264500 & -1.56344500 & -0.16264900 \\
\hline $\mathrm{H}$ & -1.70716600 & -2.55973200 & -0.35044400 \\
\hline $\mathrm{C}$ & 1.34264500 & -1.56344500 & -0.16264900 \\
\hline $\mathrm{H}$ & 1.70716600 & -2.55973200 & -0.35044400 \\
\hline $\mathrm{N}$ & 0.00000000 & -1.30556100 & 0.23412300 \\
\hline
\end{tabular}

\section{Data for Structure 22 (planar conformer)}

$E(R B+H F-L Y P)=-434.382292497$

Sum of electronic and zero-point Energies $=-434.258850$

Imaginary frequencies $=1$

Coordinates:
C
2.08822700
0.41163200
0.00000000 


$\begin{array}{lrrr}\mathrm{C} & 1.40059700 & 1.60264200 & 0.00000000 \\ \mathrm{H} & 3.15126100 & 0.24340400 & 0.00000000 \\ \mathrm{H} & 1.78642500 & 2.60737000 & 0.00000000 \\ \mathrm{C} & -1.40059700 & 1.60264200 & 0.00000000 \\ \mathrm{H} & -1.78642500 & 2.60737000 & 0.00000000 \\ \mathrm{C} & 0.68763000 & -2.01427400 & 0.00000000 \\ \mathrm{H} & 1.36483700 & -2.85077400 & 0.00000000 \\ \mathrm{C} & -0.68763000 & -2.01427400 & 0.00000000 \\ \mathrm{H} & -1.36483700 & -2.85077400 & 0.00000000 \\ \mathrm{C} & -2.08822700 & 0.41163200 & 0.00000000 \\ \mathrm{H} & -3.15126100 & 0.24340400 & 0.00000000 \\ \mathrm{~N} & 0.0000000 & 1.30728800 & 0.00000000 \\ \mathrm{~N} & -1.13214500 & -0.65364400 & 0.00000000 \\ \mathrm{~N} & 1.13214500 & -0.65364400 & 0.00000000 \\ \mathrm{C} & 0.00000000 & 0.00000000 & 0.00000000\end{array}$

\section{Data for Structure 23 (bowl conformer)}
$E(R B+H F-L Y P)=-449.529785288$
Sum of electronic and zero-point Energies $=-449.443017$
Imaginary frequencies $=0$
Coordinates:

$\begin{array}{lrrr}\text { C } & 0.00000000 & 2.03492600 & -0.14813300 \\ \mathrm{C} & 0.89475700 & 0.96437300 & 0.18821200 \\ \mathrm{C} & -1.28254900 & 0.29269600 & 0.18821200 \\ \mathrm{H} & 0.25085800 & 3.05201900 & -0.41852200 \\ \mathrm{C} & -1.76229700 & -1.01746300 & -0.14813300 \\ \mathrm{H} & -2.76855500 & -1.30876000 & -0.41852200 \\ \mathrm{C} & 1.76229700 & -1.01746300 & -0.14813300 \\ \mathrm{H} & 2.51769700 & -1.74325900 & -0.41852200 \\ \mathrm{C} & 0.38779300 & -1.25706800 & 0.18821200 \\ \mathrm{~N} & 2.06457800 & 0.31561400 & -0.15086700 \\ \mathrm{~N} & -0.75895900 & -1.94578400 & -0.15086700 \\ \mathrm{~N} & -1.30561900 & 1.63017000 & -0.15086700 \\ \mathrm{~N} & 0.00000000 & 0.00000000 & 0.52890800\end{array}$

\section{Data for Structure 23 (planar conformer)}
$E(R B+H F-L Y P)=-449.522278208$
Sum of electronic and zero-point Energies $=-449.435792$
Imaginary frequencies $=1$
Coordinates:

$\begin{array}{rrrr}\mathrm{C} & 2.03759700 & 0.47871900 & 0.00000000 \\ \mathrm{C} & 0.00000000 & 1.32768300 & 0.00000000 \\ \mathrm{~N} & 0.00000000 & 0.00000000 & 0.00000000 \\ \mathrm{C} & 1.14980700 & -0.66384100 & 0.00000000 \\ \mathrm{H} & 3.11875400 & 0.49178300 & 0.00000000\end{array}$




$\begin{array}{lrrr}\mathrm{C} & -1.43338200 & 1.52525100 & 0.00000000 \\ \mathrm{H} & -1.98527300 & 2.45502800 & 0.00000000 \\ \mathrm{C} & -0.60421500 & -2.00397000 & 0.00000000 \\ \mathrm{H} & -1.13348000 & -2.94681100 & 0.00000000 \\ \mathrm{C} & -1.14980700 & -0.66384100 & 0.00000000 \\ \mathrm{~N} & 1.34912200 & 1.67146600 & 0.00000000 \\ \mathrm{~N} & 0.77297100 & -2.00410700 & 0.00000000 \\ \mathrm{~N} & -2.12209300 & 0.33264100 & 0.00000000\end{array}$

\section{Data for Structure 24 (bowl conformer)}

$\mathrm{E}(\mathrm{RB}+\mathrm{HF}-\mathrm{LYP})=-497.587335174$
Sum of electronic and zero-point Energies $=-497.537615$
Imaginary frequencies $=0$
$\begin{array}{llrr} & & \\ \text { Coordinates: } & & & \\ \text { C } & & & \\ \text { C } & -1.10003700 & 0.63510600 & 0.22282500 \\ \text { C } & 0.00000000 & -1.27021300 & 0.22282500 \\ \text { N } & 1.10003700 & 0.63510600 & 0.22282500 \\ \text { N } & -0.67903800 & 1.87652000 & -0.20843200 \\ \text { N } & 1.96463300 & -0.35019600 & -0.20843200 \\ \text { N } & -1.28559500 & -1.52632400 & -0.20843200 \\ \text { N } & -1.96463300 & -0.35019600 & -0.20843200 \\ \text { N } & 1.28559500 & -1.52632400 & -0.20843200 \\ \text { N } & 0.67903800 & 1.87652000 & -0.20843200 \\ & 0.00000000 & 0.00000000 & 0.67761400\end{array}$

\section{Data for Structure 25 (bowl conformer)}
$E(R B+H F-L Y P)=-514.061229286$
Sum of electronic and zero-point Energies $=-514.013776$
Imaginary frequencies $=0$
Coordinates:

$\begin{array}{lrrr}\text { C } & 0.65882000 & 0.21350700 & 1.08168000 \\ \text { C } & 0.65882000 & 0.21350700 & -1.08168000 \\ \text { N } & -1.46489700 & -0.23971200 & -1.35083900 \\ \mathrm{~N} & 1.87680000 & -0.22401900 & -0.67964600 \\ \mathrm{~N} & -0.33514900 & -0.23510400 & 1.93775000 \\ \mathrm{~N} & -1.46489700 & -0.23971200 & 1.35083900 \\ \mathrm{~N} & 1.87680000 & -0.22401900 & 0.67964600 \\ \mathrm{~N} & -0.33514900 & -0.23510400 & -1.93775000 \\ \mathrm{~N} & 0.00274100 & 0.67828600 & 0.00000000 \\ \mathrm{~N} & -1.28565400 & 0.35337200 & 0.00000000\end{array}$

Data for Structure 25 (planar conformer)

$E(R B+H F-L Y P)=-514.088649209$ 
Sum of electronic and zero-point Energies $=-514.044348$

Imaginary frequencies $=0$

Coordinates:

$\begin{array}{lrrr}\mathrm{N} & 0.00000000 & 0.68124000 & 0.00000000 \\ \mathrm{C} & -1.11615300 & -0.34136900 & 0.00000000 \\ \mathrm{C} & 1.11657300 & -0.34378400 & 0.00000000 \\ \mathrm{~N} & -3.34774700 & 0.58739300 & 0.00000000 \\ \mathrm{~N} & -0.64536200 & -1.62417300 & 0.00000000 \\ \mathrm{~N} & 2.34271300 & 0.05595600 & 0.00000000 \\ \mathrm{~N} & -2.34202700 & 0.05975700 & 0.00000000 \\ \mathrm{~N} & 0.64310700 & -1.62544600 & 0.00000000 \\ \mathrm{~N} & 3.34841400 & 0.58387100 & 0.00000000 \\ \mathrm{~N} & 0.00054300 & 1.86867500 & 0.00000000\end{array}$

\section{Data for Structure 26 (bowl conformer)}

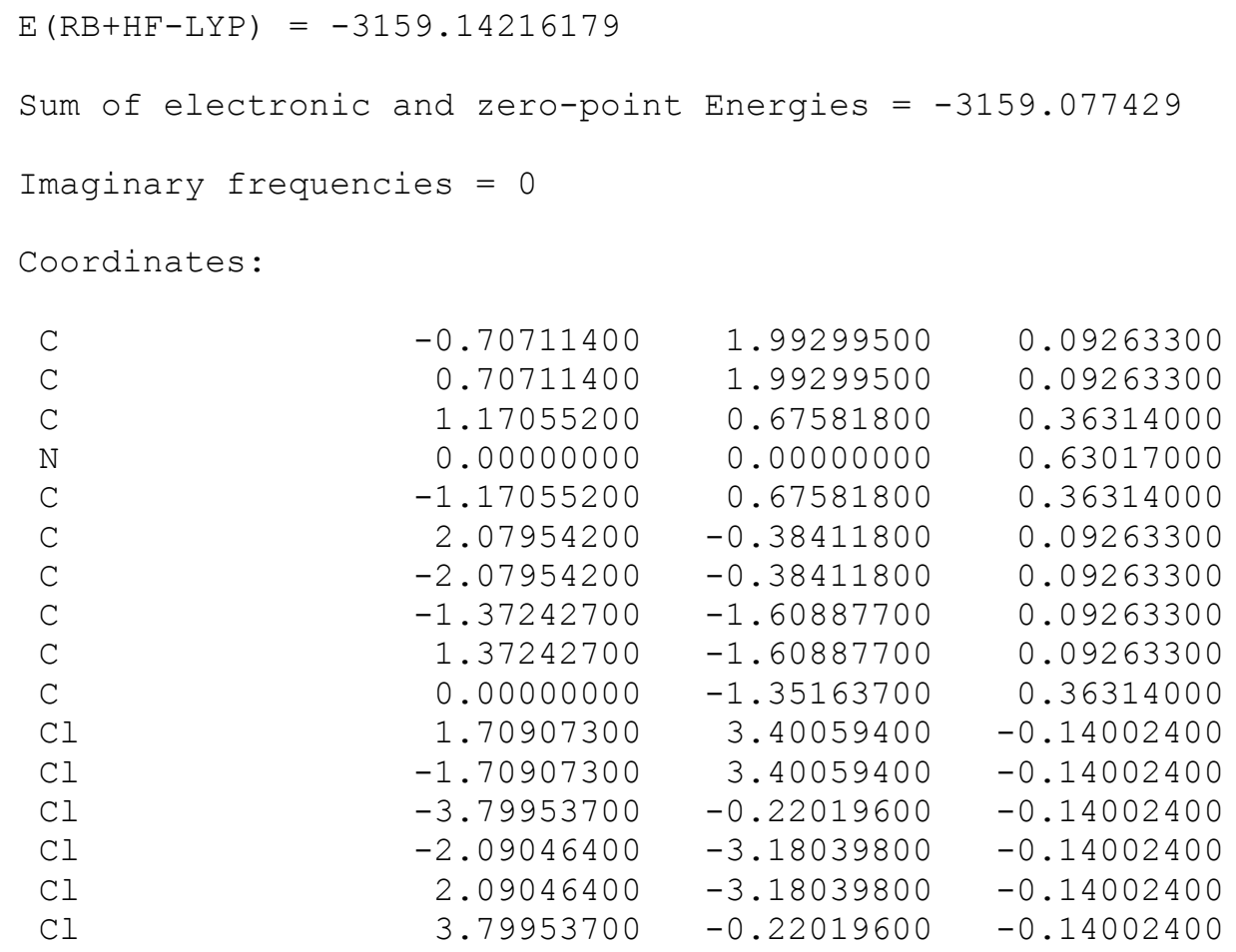

\section{Data for Structure 26 (planar conformer)}

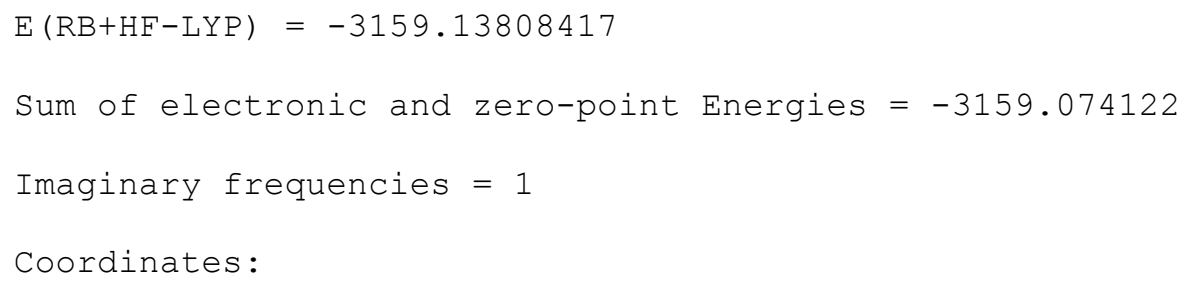




$\begin{array}{lrrr}\text { C } & 2.11226800 & 0.39789000 & 0.00000000 \\ C & 1.40071700 & 1.63033200 & 0.00000000 \\ C & 0.00000000 & 1.35690800 & 0.00000000 \\ \text { N } & 0.00000000 & 0.00000000 & 0.00000000 \\ C & 1.17511600 & -0.67845400 & 0.00000000 \\ C & -1.40071700 & 1.63033200 & 0.00000000 \\ C & 0.71155100 & -2.02822300 & 0.00000000 \\ C & -0.71155100 & -2.02822300 & 0.00000000 \\ C & -2.11226800 & 0.39789000 & 0.00000000 \\ C & -1.17511600 & -0.67845400 & 0.00000000 \\ C 1 & -2.13798200 & 3.20923200 & 0.00000000 \\ C 1 & 2.13798200 & 3.20923200 & 0.00000000 \\ C 1 & 3.84826700 & 0.24693100 & 0.00000000 \\ C 1 & 1.71028600 & -3.45616300 & 0.00000000 \\ C 1 & -1.71028600 & -3.45616300 & 0.00000000 \\ C 1 & -3.84826700 & 0.24693100 & 0.00000000\end{array}$

\section{Data for Structure 27 (bowl conformer)}

\begin{tabular}{|c|c|c|c|}
\hline \multicolumn{4}{|c|}{ Sum of electronic and zero-point Energies $=-954.961052$} \\
\hline \multicolumn{4}{|c|}{ Imaginary frequencies $=0$} \\
\hline \multicolumn{4}{|c|}{ Coordinates: } \\
\hline $\mathrm{C}$ & -0.72315100 & 2.02840800 & 0.06077700 \\
\hline $\mathrm{C}$ & 0.72315100 & 2.02840800 & 0.06077700 \\
\hline $\mathrm{C}$ & 1.16561700 & 0.67296900 & 0.18515000 \\
\hline $\mathrm{N}$ & 0.00000000 & 0.00000000 & 0.31326400 \\
\hline $\mathrm{C}$ & -1.16561700 & 0.67296900 & 0.18515000 \\
\hline $\mathrm{C}$ & 2.11822800 & -0.38793700 & 0.06077700 \\
\hline $\mathrm{C}$ & -2.11822800 & -0.38793700 & 0.06077700 \\
\hline $\mathrm{C}$ & -1.39507700 & -1.64047100 & 0.06077700 \\
\hline $\mathrm{C}$ & 1.39507700 & -1.64047100 & 0.06077700 \\
\hline $\mathrm{C}$ & 0.00000000 & -1.34593800 & 0.18515000 \\
\hline $\mathrm{C}$ & 1.53481400 & 3.17871000 & -0.04925500 \\
\hline $\mathrm{N}$ & 2.20020800 & 4.12154400 & -0.14143700 \\
\hline $\mathrm{C}$ & 3.52025100 & -0.26016800 & -0.04925500 \\
\hline $\mathrm{N}$ & 4.66946600 & -0.15533600 & -0.14143700 \\
\hline $\mathrm{C}$ & 1.98543700 & -2.91854300 & -0.04925500 \\
\hline $\mathrm{N}$ & 2.46925800 & -3.96620800 & -0.14143700 \\
\hline $\mathrm{C}$ & -1.98543700 & -2.91854300 & -0.04925500 \\
\hline $\mathrm{N}$ & -2.46925800 & -3.96620800 & -0.14143700 \\
\hline $\mathrm{C}$ & -3.52025100 & -0.26016800 & -0.04925500 \\
\hline $\mathrm{N}$ & -4.66946600 & -0.15533600 & -0.14143700 \\
\hline $\mathrm{C}$ & -1.53481400 & 3.17871000 & -0.04925500 \\
\hline $\mathrm{N}$ & -2.20020800 & 4.12154400 & -0.14143700 \\
\hline
\end{tabular}

\section{Data for Structure 27 (planar conformer)}

$E(R B+H F-L Y P)=-955.074435943$

Sum of electronic and zero-point Energies $=-954.961554$ 
Imaginary frequencies $=1$

Coordinates:

\begin{tabular}{|c|c|c|c|}
\hline C & 2.12555400 & 0.39112700 & 0.00000000 \\
\hline $\mathrm{C}$ & 1.40150300 & 1.64522000 & 0.00000000 \\
\hline $\mathrm{C}$ & 0.00000000 & 1.34766900 & 0.00000000 \\
\hline $\mathrm{N}$ & 0.00000000 & 0.00000000 & 0.00000000 \\
\hline $\mathrm{C}$ & 1.16711600 & -0.67383500 & 0.00000000 \\
\hline $\mathrm{C}$ & -1.40150300 & 1.64522000 & 0.00000000 \\
\hline $\mathrm{C}$ & 0.72405100 & -2.03634700 & 0.00000000 \\
\hline $\mathrm{C}$ & -0.72405100 & -2.03634700 & 0.00000000 \\
\hline $\mathrm{C}$ & -2.12555400 & 0.39112700 & 0.00000000 \\
\hline $\mathrm{C}$ & -1.16711600 & -0.67383500 & 0.00000000 \\
\hline $\mathrm{C}$ & 1.99716500 & 2.92528100 & 0.00000000 \\
\hline $\mathrm{N}$ & 2.48489900 & 3.97522900 & 0.00000000 \\
\hline $\mathrm{C}$ & 3.53195000 & 0.26695500 & 0.00000000 \\
\hline $\mathrm{N}$ & 4.68509900 & 0.16437100 & 0.00000000 \\
\hline $\mathrm{C}$ & 1.53478500 & -3.19223600 & 0.00000000 \\
\hline $\mathrm{N}$ & 2.20020000 & -4.13960000 & 0.00000000 \\
\hline $\mathrm{C}$ & -1.53478500 & -3.19223600 & 0.00000000 \\
\hline $\mathrm{N}$ & -2.20020000 & -4.13960000 & 0.00000000 \\
\hline $\mathrm{C}$ & -3.53195000 & 0.26695500 & 0.00000000 \\
\hline $\mathrm{N}$ & -4.68509900 & 0.16437100 & 0.00000000 \\
\hline $\mathrm{C}$ & -1.99716500 & 2.92528100 & 0.00000000 \\
\hline $\mathrm{N}$ & -2.48489900 & 3.97522900 & 0.00000000 \\
\hline
\end{tabular}

\section{Data for Structure 28 (bowl conformer)}

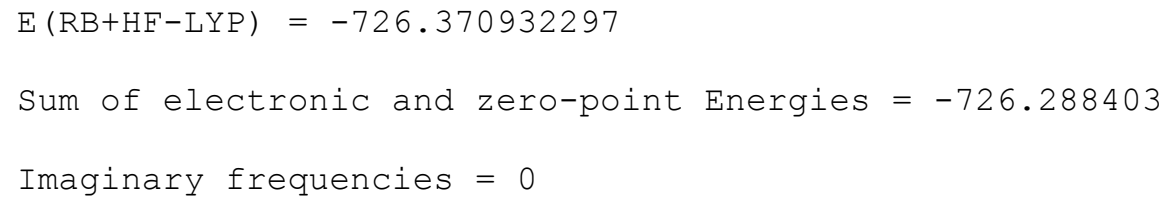

$\begin{array}{lrrr}\text { C } & -2.01485600 & 0.31632700 & 0.07878600 \\ \text { C } & -0.80275600 & 1.03728200 & 0.39610000 \\ \text { C } & -0.49693400 & -1.21384800 & 0.39610000 \\ \text { C } & 0.73348000 & -1.90308000 & 0.07878600 \\ C & 1.28137600 & 1.58675300 & 0.07878600 \\ \text { C } & 1.29969000 & 0.17656600 & 0.39610000 \\ N & 0.00000000 & 2.08590400 & 0.06652200 \\ N & 1.80644600 & -1.04295200 & 0.06652200 \\ N & -1.80644600 & -1.04295200 & 0.06652200 \\ N & 0.00000000 & 0.00000000 & 0.73856400 \\ C & -3.28078900 & 0.86629900 & -0.24626100 \\ N & -4.30606900 & 1.33568100 & -0.50867500 \\ C & 2.39063100 & 2.40809700 & -0.24626100 \\ N & 3.30976800 & 3.06132400 & -0.50867500 \\ C & 0.89015800 & -3.27439600 & -0.24626100 \\ N & 0.99630100 & -4.39700500 & -0.50867500\end{array}$

\section{Data for Structure 28 (planar conformer)}


$E(R B+H F-L Y P)=-726.364117347$

Sum of electronic and zero-point Energies $=-726.281933$

Imaginary frequencies $=1$

Coordinates:

\begin{tabular}{|c|c|c|c|}
\hline $\mathrm{C}$ & 2.03352000 & 0.49628100 & 0.00000000 \\
\hline $\mathrm{C}$ & 0.00000000 & 1.32580800 & 0.00000000 \\
\hline $\mathrm{N}$ & 0.00000000 & 0.00000000 & 0.00000000 \\
\hline $\mathrm{C}$ & 1.14818300 & -0.66290400 & 0.00000000 \\
\hline $\mathrm{C}$ & -1.44655200 & 1.51293900 & 0.00000000 \\
\hline $\mathrm{C}$ & -0.58696800 & -2.00922000 & 0.00000000 \\
\hline $\mathrm{C}$ & -1.14818300 & -0.66290400 & 0.00000000 \\
\hline $\mathrm{N}$ & 1.32525800 & 1.68505200 & 0.00000000 \\
\hline $\mathrm{N}$ & 0.79666900 & -1.99023400 & 0.00000000 \\
\hline $\mathrm{N}$ & -2.12192700 & 0.30518100 & 0.00000000 \\
\hline $\mathrm{C}$ & 3.45056400 & 0.50123000 & 0.00000000 \\
\hline $\mathrm{N}$ & 4.60811000 & 0.47770500 & 0.00000000 \\
\hline $\mathrm{C}$ & -2.15936000 & 2.73766100 & 0.00000000 \\
\hline $\mathrm{N}$ & -2.71776000 & 3.75188800 & 0.00000000 \\
\hline $\mathrm{C}$ & -1.29120400 & -3.23889100 & 0.00000000 \\
\hline $\mathrm{N}$ & -1.89035100 & -4.22959300 & 0.00000000 \\
\hline
\end{tabular}

\section{Data for Structure 29}

$E(R B+H F-L Y P)=-441.204320895$

Sum of electronic and zero-point Energies $=-441.042539$

Imaginary frequencies $=0$

Coordinates:

$\begin{array}{lrrr}\mathrm{C} & -2.06795000 & 0.36979700 & -0.40358500 \\ \mathrm{C} & -1.35422900 & 1.60599900 & -0.40358500 \\ \mathrm{C} & 0.00000000 & 1.38665600 & -0.10605000 \\ \mathrm{~N} & 0.00000000 & 0.00000000 & 0.37891200 \\ \mathrm{C} & -1.20087900 & -0.69332800 & -0.10605000 \\ \mathrm{H} & -3.10037100 & 0.27097500 & -0.70651400 \\ \mathrm{H} & -1.78485600 & 2.54951200 & -0.70651400 \\ \mathrm{C} & 1.35422900 & 1.60599900 & -0.40358500 \\ \mathrm{H} & 1.78485600 & 2.54951200 & -0.70651400 \\ \mathrm{C} & -0.71372100 & -1.97579600 & -0.40358500 \\ \mathrm{H} & -1.31551400 & -2.82048700 & -0.70651400 \\ \mathrm{C} & 0.71372100 & -1.97579600 & -0.40358500 \\ \mathrm{H} & 1.31551400 & -2.82048700 & -0.70651400 \\ \mathrm{C} & 2.06795000 & 0.36979700 & -0.40358500 \\ \mathrm{H} & 3.10037100 & 0.27097500 & -0.70651400 \\ \mathrm{C} & 1.20087900 & -0.69332800 & -0.10605000 \\ \mathrm{C} & 0.00000000 & 0.00000000 & 1.89269900 \\ \mathrm{H} & 0.89820200 & 0.51857700 & 2.22282200 \\ \mathrm{H} & 0.00000000 & -1.03715500 & 2.22282200 \\ \mathrm{H} & -0.89820200 & 0.51857700 & 2.22282200\end{array}$


Data for Structure 30

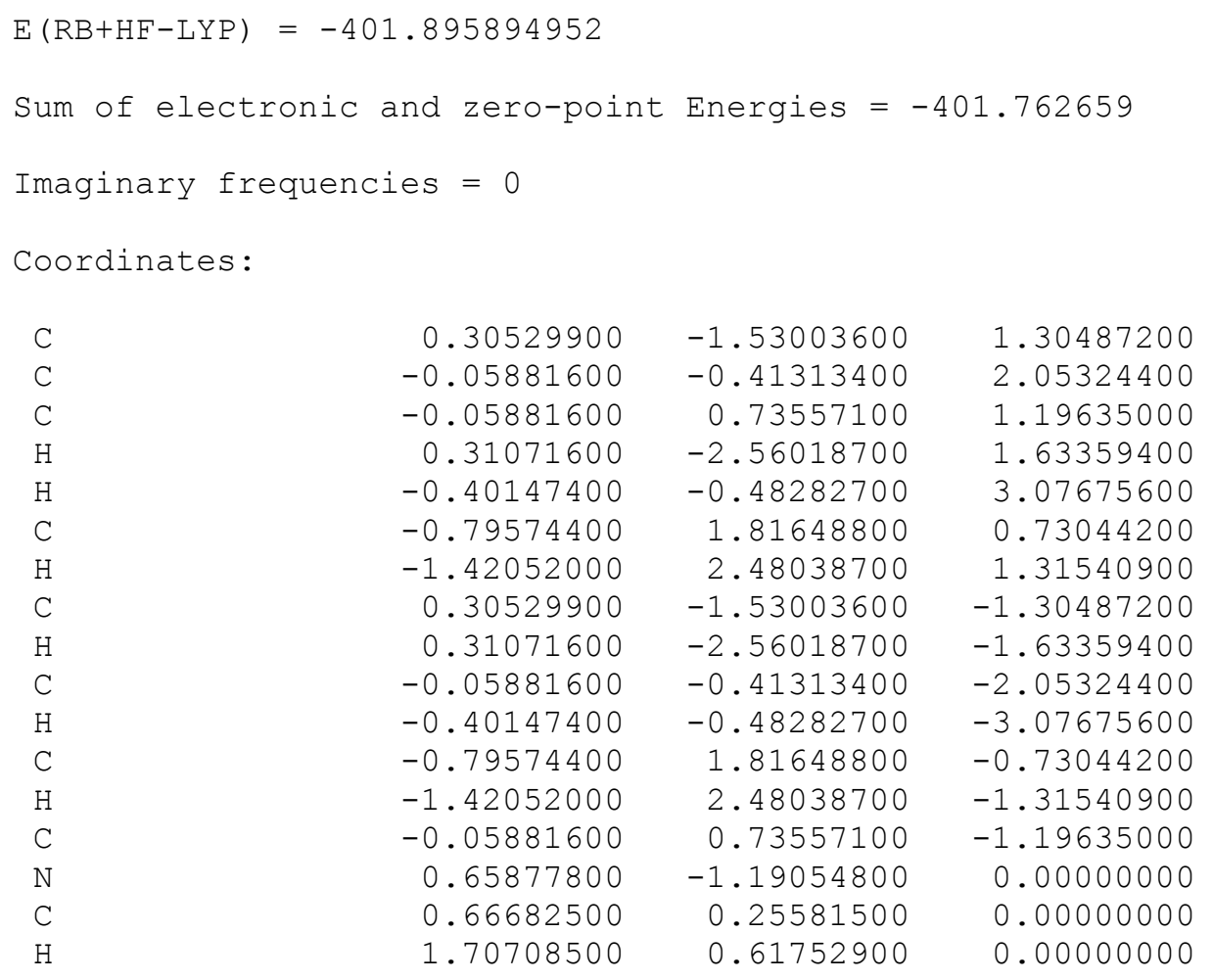

\section{Data for Structure 31}

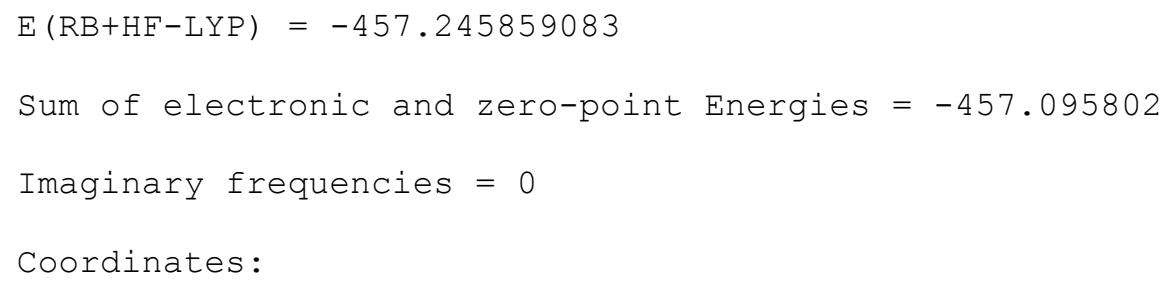

$\begin{array}{lrrr}\mathrm{C} & -0.86469300 & -1.88105800 & -0.40181000 \\ \mathrm{C} & -1.88313400 & -0.86899600 & -0.41680000 \\ \mathrm{C} & -1.30894300 & 0.36799700 & -0.11335300 \\ \mathrm{~N} & 0.01357000 & 0.00402900 & 0.41581200 \\ \mathrm{C} & 0.37765700 & -1.33024900 & -0.06808200 \\ \mathrm{H} & -1.02982300 & -2.89691800 & -0.73188100 \\ \mathrm{H} & -2.89186600 & -1.02665100 & -0.76760000 \\ \mathrm{C} & 1.74215200 & -1.16641800 & -0.39463700 \\ \mathrm{H} & 2.41207300 & -1.95519500 & -0.70608000 \\ \mathrm{C} & 2.07773900 & 0.21279900 & -0.42587300 \\ \mathrm{H} & 3.03573500 & 0.59090400 & -0.75273700 \\ \mathrm{C} & 0.06311300 & 2.03796200 & -0.45380600 \\ \mathrm{H} & 0.32568700 & 3.03245100 & -0.78327300 \\ \mathrm{C} & 0.94659600 & 1.00062000 & -0.11241700 \\ \mathrm{C} & -0.00241000 & 0.04099800 & 1.92512000 \\ \mathrm{H} & -0.31240200 & 1.03904900 & 2.23018500 \\ \mathrm{H} & 1.00615800 & -0.17675100 & 2.27172900 \\ \mathrm{H} & -0.70954300 & -0.71207800 & 2.26836100\end{array}$




\section{Data for Structure 32}

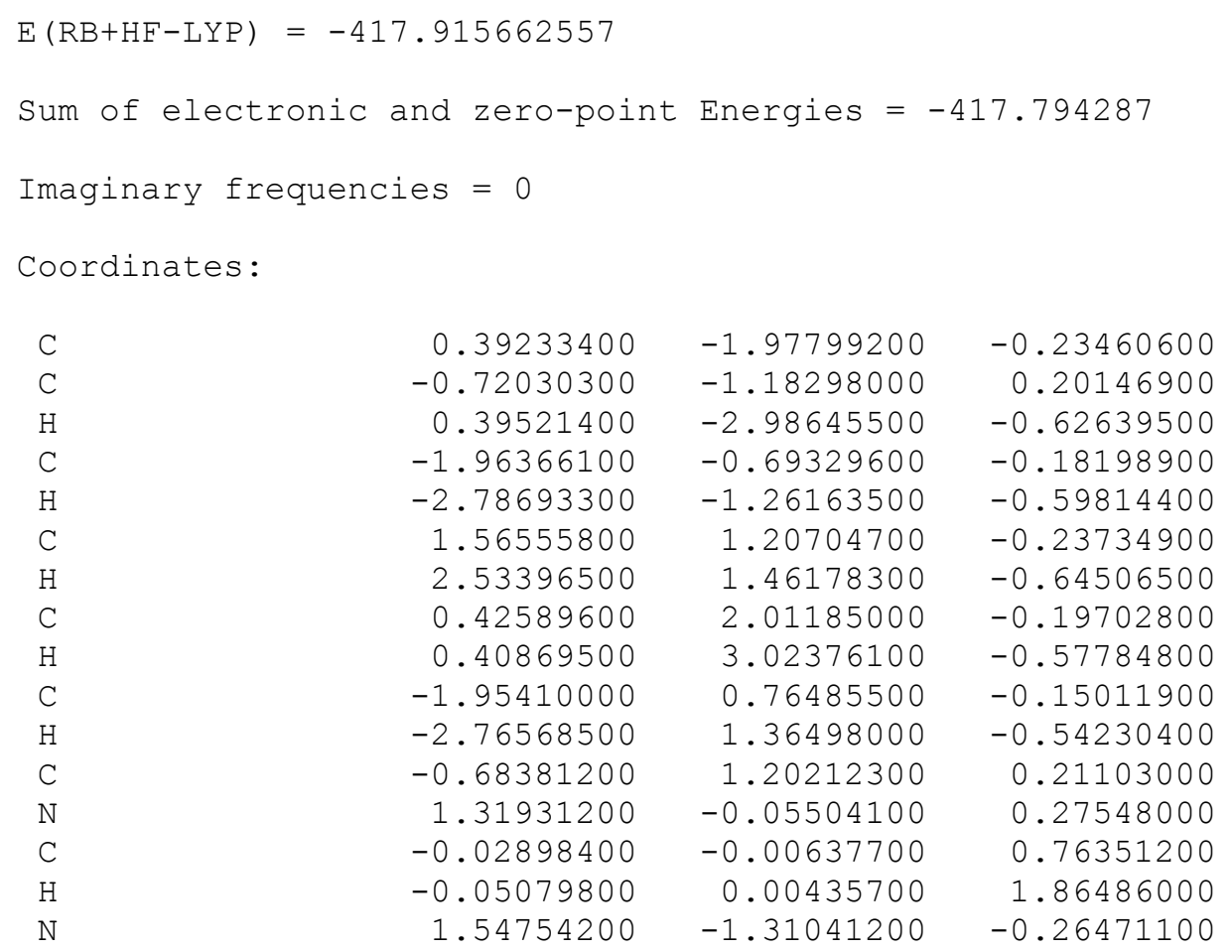

\section{Data for Structure 33}

$\mathrm{E}(\mathrm{RB}+\mathrm{HF}-\mathrm{LYP})=-417.936455478$

Sum of electronic and zero-point Energies $=-417.814925$

Imaginary frequencies $=0$

Coordinates:

$\begin{array}{lrrr}\text { C } & -0.65040800 & 1.85229800 & -0.24621100 \\ \mathrm{C} & 1.14193900 & 0.73200300 & 0.20380700 \\ \mathrm{H} & -1.29778700 & 2.64132900 & -0.60468000 \\ \mathrm{C} & 2.05854600 & -0.21896000 & -0.19284300 \\ \mathrm{H} & 3.02047000 & -0.04945100 & -0.65886000 \\ \mathrm{C} & -1.96268900 & -0.41741400 & -0.22529100 \\ \mathrm{H} & -2.97007600 & -0.24638900 & -0.57873100 \\ \mathrm{C} & -1.28454800 & -1.62633300 & -0.18961900 \\ \mathrm{H} & -1.71357900 & -2.54677800 & -0.56287500 \\ \mathrm{C} & 1.39401400 & -1.53007600 & -0.17591700 \\ \mathrm{H} & 1.84923500 & -2.41621500 & -0.60154800 \\ \mathrm{C} & 0.07454400 & -1.38353700 & 0.21817800 \\ \mathrm{~N} & -1.17171600 & 0.64695500 & 0.24552500 \\ \mathrm{C} & 0.00667900 & -0.00967800 & 0.77608000 \\ \mathrm{H} & -0.01883600 & -0.01809200 & 1.87540300 \\ \mathrm{~N} & 0.66630300 & 1.95958500 & -0.22806900\end{array}$


Data for Structure 34

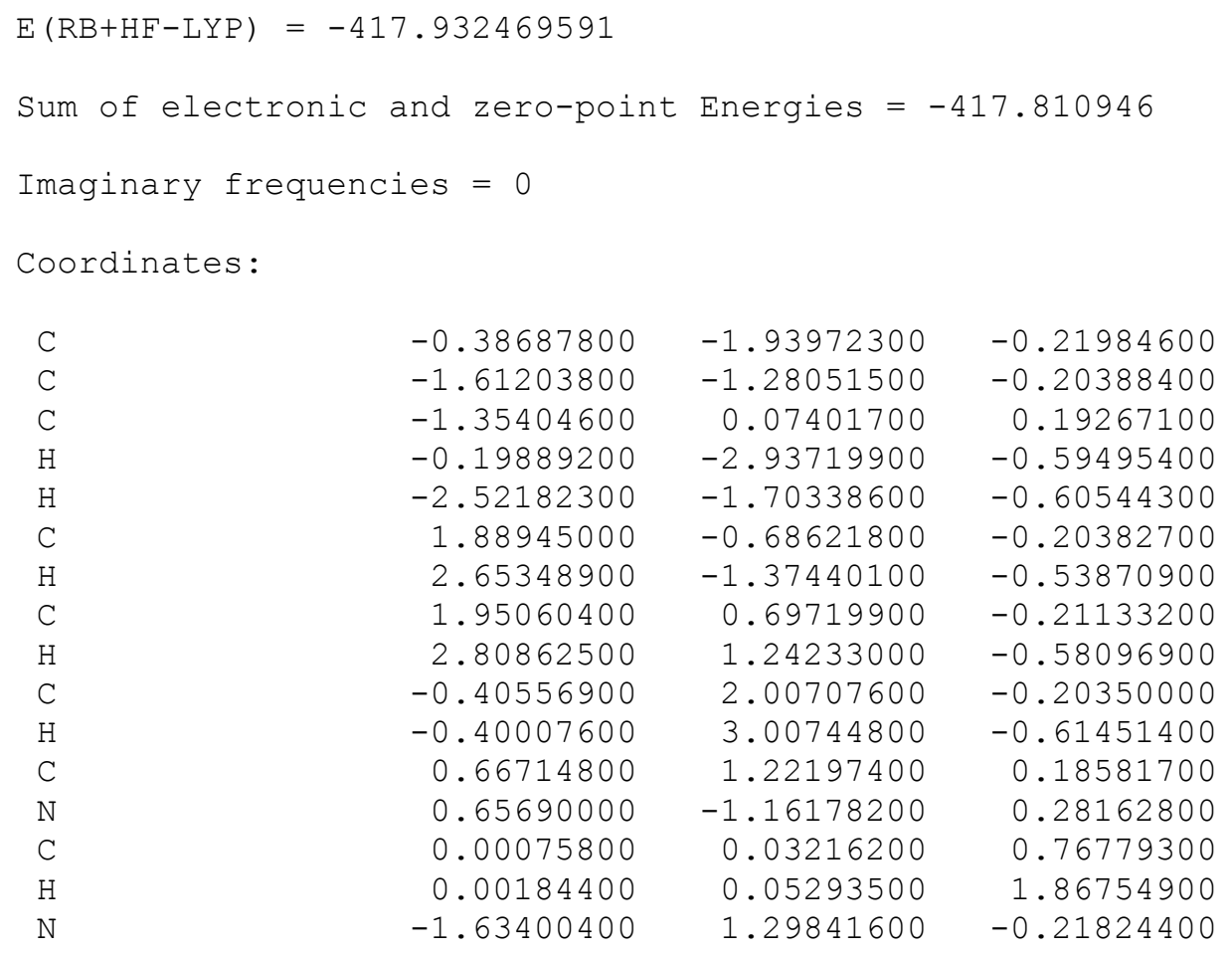

Data for Structure 35

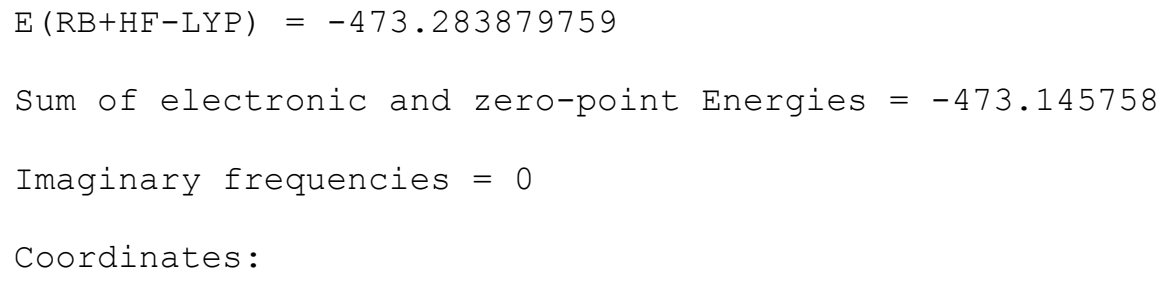

$\begin{array}{lrrr}\mathrm{C} & -1.64598700 & -1.23282200 & -0.42196600 \\ \mathrm{C} & -2.06630400 & 0.13097800 & -0.40464200 \\ \mathrm{C} & -0.96761300 & 0.94634700 & -0.07853700 \\ \mathrm{~N} & 0.00532400 & -0.01304900 & 0.45355700 \\ \mathrm{C} & -0.28636800 & -1.34587100 & -0.06895500 \\ \mathrm{H} & -2.26866600 & -2.04172000 & -0.77796600 \\ \mathrm{H} & -3.02350700 & 0.47693700 & -0.76511800 \\ \mathrm{C} & 1.01234500 & -1.76517000 & -0.44666700 \\ \mathrm{H} & 1.30172000 & -2.75021400 & -0.78356900 \\ \mathrm{C} & 1.05312400 & 1.71192400 & -0.47271300 \\ \mathrm{H} & 1.76436900 & 2.43034600 & -0.84962600 \\ \mathrm{C} & 1.29477600 & 0.38016400 & -0.12078200 \\ \mathrm{C} & 0.04336500 & 0.00796600 & 1.95966500 \\ \mathrm{H} & 0.22952400 & 1.03295700 & 2.27639900 \\ \mathrm{H} & 0.84886800 & -0.64972800 & 2.28126000 \\ \mathrm{H} & -0.91959600 & -0.34729700 & 2.32226000 \\ \mathrm{~N} & -0.29648600 & 2.02732400 & -0.45555900 \\ \mathrm{~N} & 1.92591400 & -0.75033000 & -0.48029200\end{array}$




\section{Data for Structure 36 (bowl conformer)}

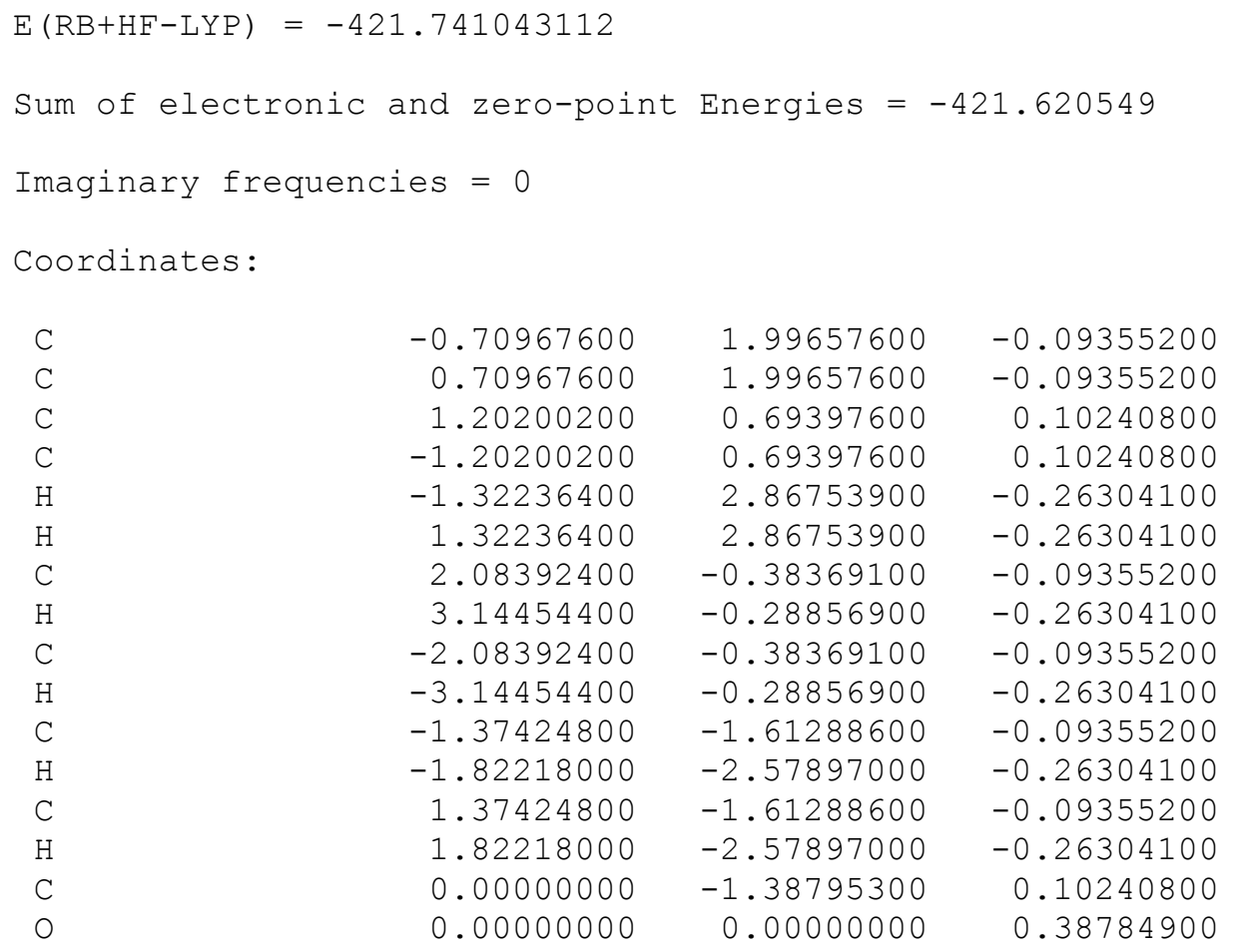

Data for Structure 36 (planar conformer)

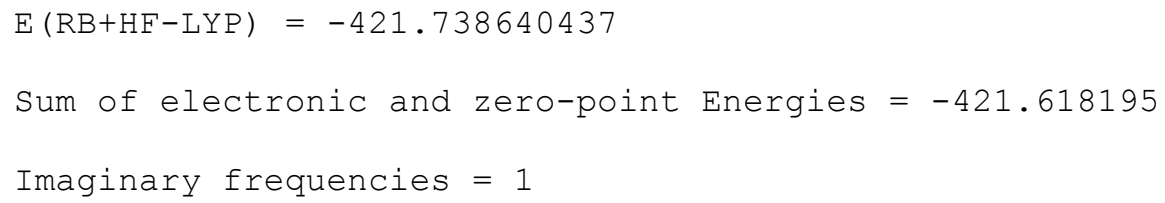

$\begin{array}{lrrr}\text { C } & 2.10507500 & 0.39324000 & 0.00000000 \\ \mathrm{C} & 1.39309300 & 1.62642800 & 0.00000000 \\ \mathrm{C} & 0.00000000 & 1.39334100 & 0.00000000 \\ \mathrm{C} & 1.20666900 & -0.69667000 & 0.00000000 \\ \mathrm{H} & 3.17952200 & 0.30798300 & 0.00000000 \\ \mathrm{H} & 1.85648200 & 2.59955600 & 0.00000000 \\ \mathrm{C} & -1.39309300 & 1.62642800 & 0.00000000 \\ \mathrm{H} & -1.85648200 & 2.59955600 & 0.00000000 \\ \mathrm{C} & 0.71198200 & -2.01966800 & 0.00000000 \\ \mathrm{H} & 1.32304000 & -2.90753800 & 0.00000000 \\ \mathrm{C} & -0.71198200 & -2.01966800 & 0.00000000 \\ \mathrm{H} & -1.32304000 & -2.90753800 & 0.00000000 \\ \mathrm{C} & -2.10507500 & 0.39324000 & 0.00000000 \\ \mathrm{H} & -3.17952200 & 0.30798300 & 0.00000000 \\ \mathrm{C} & -1.20666900 & -0.69667000 & 0.00000000 \\ \mathrm{O} & 0.00000000 & 0.00000000 & 0.00000000\end{array}$


Data for Structure 37 (bowl conformer)

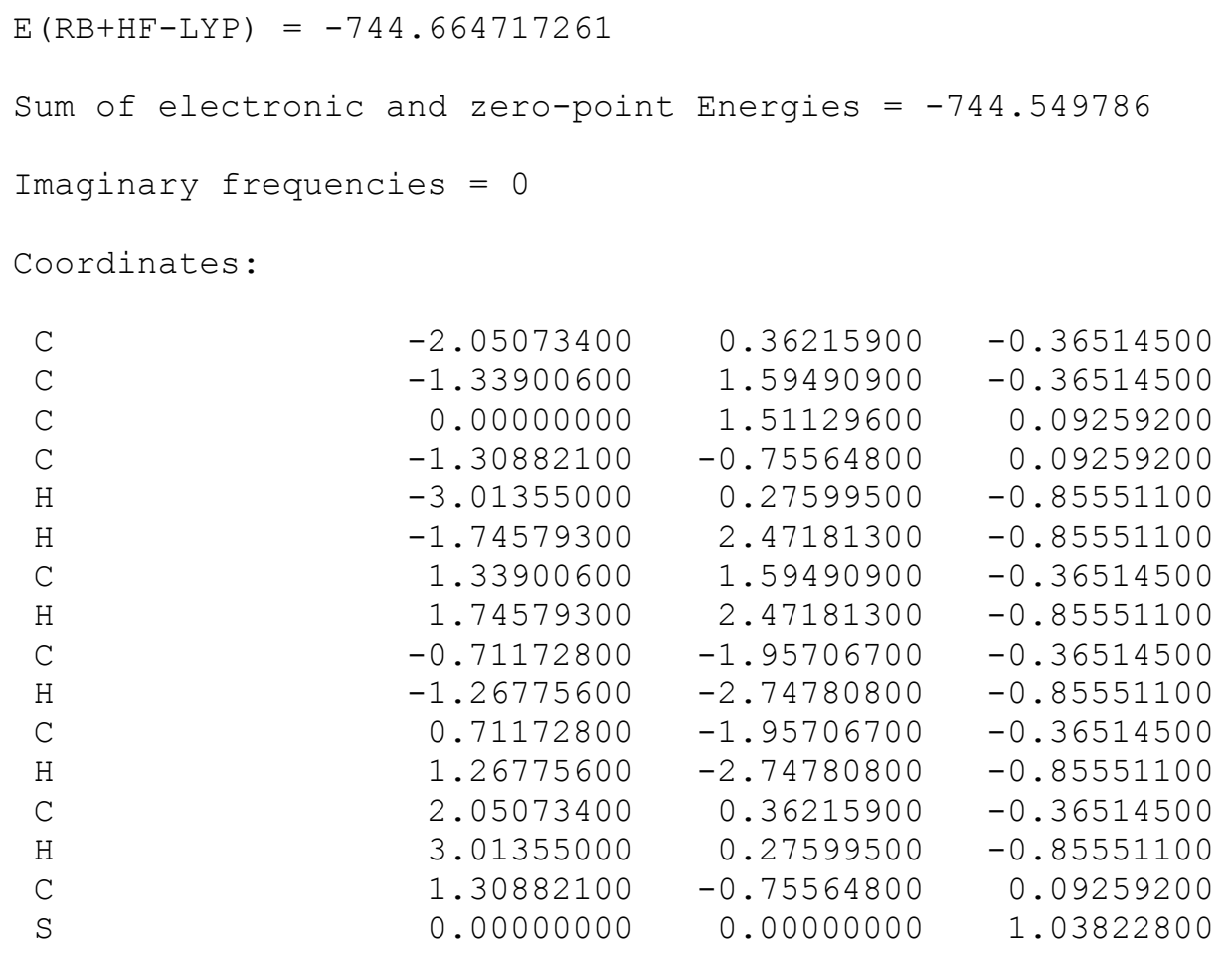

Data for Structure 37 (planar conformer)

$\begin{array}{llll}\mathrm{E}(\mathrm{RB}+\mathrm{HF}-\mathrm{LYP}) \quad=-744.535523970 & \\ \text { Sum of electronic and zero-point Energies }=-744.421719 \\ \text { Imaginary frequencies }=1 & & & \\ & & & \\ \text { Coordinates: } & & & \\ & & & \\ \text { C } & 2.20926600 & 0.44210500 & 0.00000000 \\ \text { C } & 1.48750700 & 1.69222800 & 0.00000000 \\ \text { C } & 0.00000000 & 1.60530700 & 0.00000000 \\ \text { C } & 1.39023700 & -0.80265400 & 0.00000000 \\ \text { H } & 3.28972500 & 0.44169800 & 0.00000000 \\ \text { H } & 2.02738400 & 2.62813700 & 0.00000000 \\ \text { C } & -1.48750700 & 1.69222800 & 0.00000000 \\ \text { H } & -2.02738400 & 2.62813700 & 0.00000000 \\ \text { C } & 0.72175900 & -2.13433300 & 0.00000000 \\ \text { H } & 1.26234100 & -3.06983400 & 0.00000000 \\ \text { C } & -0.72175900 & -2.13433300 & 0.00000000 \\ \text { H } & -1.26234100 & -3.06983400 & 0.00000000 \\ \text { C } & -2.20926600 & 0.44210500 & 0.00000000 \\ \text { H } & -3.28972500 & 0.44169800 & 0.00000000 \\ \text { C } & -1.39023700 & -0.80265400 & 0.00000000 \\ \text { S } & 0.00000000 & 0.00000000 & 0.00000000\end{array}$

